\title{
A POSTERIORI ERROR ESTIMATOR AND ERROR CONTROL FOR CONTACT PROBLEMS
}

\author{
ALEXANDER WEISS AND BARBARA I. WOHLMUTH
}

\begin{abstract}
In this paper, we consider two error estimators for one-body contact problems. The first error estimator is defined in terms of $H$ (div)-conforming stress approximations and equilibrated fluxes while the second is a standard edge-based residual error estimator without any modification with respect to the contact. We show reliability and efficiency for both estimators. Moreover, the error is bounded by the first estimator with a constant one plus a higher order data oscillation term plus a term arising from the contact that is shown numerically to be of higher order. The second estimator is used in a control-based AFEM refinement strategy, and the decay of the error in the energy is shown. Several numerical tests demonstrate the performance of both estimators.
\end{abstract}

\section{INTRODUCTION}

Finite element methods are commonly used in the numerical simulation of many engineering applications. Adaptive techniques based on a posteriori error estimators have become an indispensable tool and are well established for such methods; see [2, 27, 55] and the references therein. Error estimators have also been successfully used in the field of nonconforming discretizations [1, 19, 20, 24 and for variational inequalities [3, 13, 26] such as obstacle problems; see [14, 32, 34, 53] and for contact problems, see [11, 12, 18, 22, 31, 41, 61].

Recently, error control strategies, commonly known as AFEM refinement strategies, have been developed, e.g., in [5, 21, 25, 42, 45, 46]. Here, special refinement rules in combination with a control over the data oscillation terms lead to a guaranteed error decay. Moreover, optimal convergence under mild regularity assumptions has been stated in [10, 51, 52, These control-based strategies have also been applied to obstacle problems [15]. Here, a decay in the energy can be achieved instead of the error decay.

Usually upper and lower bounds are proved in order to guarantee the reliability and the efficiency of the proposed estimator. Most of the existing approaches involve constants depending on the shape regularity of the elements and/or of the jumps in the coefficients, but these dependences are often not given explicitly. However, in a recent paper [44] an error estimator for the Lamé equation based on $H$ (div)-conforming approximations for the stress and on equilibrated fluxes has

Received by the editor July 17, 2007 and, in revised form, June 2, 2008.

2000 Mathematics Subject Classification. Primary 65N30, 65N15, 65N50.

Key words and phrases. Equilibrated fluxes, Lagrange multipliers, a posteriori error estimates, contact problems.

This work was supported in part by the Deutsche Forschungsgemeinschaft, SFB 404, B8.

(C)2009 American Mathematical Society Reverts to public domain 28 years from publication 
been introduced which guarantees an upper bound with constant one up to data oscillation terms.

In this paper, we adapt this error estimator to one-body contact problems. Then, the error is bounded by the same error estimator with a constant one plus an extra term arising from the contact which proves numerically to be of higher order. Moreover it is shown that the extra term can be neglected without losing the reliability of the error estimator. Using a similar technique, also a new upper bound for the error in the Lagrange multiplier is established.

We also define a control-based refinement strategy. Here a standard edge-based residual error estimator is used in combination with the well-known AFEM marking and refinement technique 42. Even though the contact stress is not used in the definition of this error estimator, reliability and efficiency for one-body contact problems are established. Moreover, we show a strict energy decay when using the AFEM technique.

The rest of the paper is organized as follows: In Section 2, we recall the contact problem and its discretization. Section 3 is devoted to the introduction of the estimator and the proofs of the upper and lower bounds. In Section 4, we present some numerical examples that demonstrate the usage of our error estimator for contact problems. A new upper bound for the Lagrange multiplier is derived in Section 5. The last two sections are dedicated to an AFEM energy control strategy. In Section 6, the strategy based on an edge-based error estimator is presented and energy decay is shown. Numerical examples for this strategy and comparisons between the two error estimators are given in Section 7 .

\section{Contact PRoblem AND Discretization}

In this section, we state the setting of a contact problem between an elastic body and a rigid obstacle without friction and its discretization. The body in its reference configuration is given by $\Omega \subset \mathbb{R}^{2}$. We assume the boundary $\partial \Omega$ to be divided into three open disjoint measurable parts $\Gamma_{D}, \Gamma_{N}, \Gamma_{C}$ with meas $\left(\Gamma_{D}\right)>0$. Furthermore, we assume that $\bar{\Gamma}_{C}$ is a compact subset of $\partial \Omega \backslash \bar{\Gamma}_{D}$. We denote the outer unit normal vector on the boundary $\partial \Omega$ by $\mathbf{n}$. On the contact zone the normal vector $\mathbf{n}$ is assumed to be constant. We impose Dirichlet conditions on $\Gamma_{D}$ and homogeneous Neumann data on $\Gamma_{N}$. Moreover, we denote volume forces by $\mathbf{f} \in\left[L^{2}(\Omega)\right]^{2}$. We consider a homogeneous isotropic linearized Saint VenantKirchhoff material, where the stress tensor is given in terms of Hooke's tensor $\mathcal{C}$ by

$$
\boldsymbol{\sigma}(\mathbf{v}):=\lambda \operatorname{tr}(\varepsilon(\mathbf{v})) \mathbf{I d}+2 \mu \varepsilon(\mathbf{v})=: \mathcal{C} \varepsilon(\mathbf{v}),
$$

and the linearized strain tensor is defined by $\varepsilon(\mathbf{v}):=\frac{1}{2}\left(\nabla \mathbf{v}+(\nabla \mathbf{v})^{\top}\right)$. The positive material parameters $\lambda$ and $\mu$ are the Lamé parameters and are assumed to be constant. Moreover, tr denotes the matrix trace operator and Id the identity matrix in $\mathbb{R}^{2}$. The elastic equilibrium condition can be written as

$$
\begin{array}{rlll}
-\operatorname{div} \boldsymbol{\sigma}(\mathbf{u}) & =\mathbf{f} & \text { in } & \Omega, \\
\mathbf{u} & =\mathbf{u}_{D} & \text { on } & \Gamma_{D}, \\
\boldsymbol{\sigma}(\mathbf{u}) \mathbf{n} & =\mathbf{0} & \text { on } & \Gamma_{N} .
\end{array}
$$

In addition to (2.1), we have to satisfy the linearized nonpenetration condition. Taking into account that we do not consider friction, we find on $\Gamma_{C}$ the following 
equality and inequality constraints:

$$
\begin{gathered}
\mathbf{u}_{n} \leq 0, \quad \sigma_{n} \leq 0, \quad \sigma_{n} \mathbf{u}_{n}=0, \\
\boldsymbol{\sigma}_{t}=0,
\end{gathered}
$$

where $\sigma_{n}:=\mathbf{n} \cdot \boldsymbol{\sigma}(\mathbf{u}) \mathbf{n}$ and $\boldsymbol{\sigma}_{t}=\boldsymbol{\sigma}(\mathbf{u}) \mathbf{n}-\sigma_{n} \mathbf{n}$ are the normal and tangential components, respectively, of the boundary stress, and $\mathbf{u}_{n}:=\mathbf{u} \cdot \mathbf{n}$ stands for the displacement in the normal direction. Here, we have been assuming for simplicity that the body in its reference configuration is in contact with the rigid obstacle on $\Gamma_{C}$ and that there is no gap.

2.1. Weak formulation. It is well known that the solution of (2.1)-(2.3) can be rewritten as a solution of a constrained minimization problem or equivalently as a variational inequality on a convex set; see, e.g., [37. To give the variational formulation of problem (2.1) with the nonpenetration condition (2.2) and the condition of no friction (2.3), we introduce the space $\boldsymbol{V}:=\left[H^{1}(\Omega)\right]^{2}$, equipped with the $H^{1}$-norm $\|\mathbf{v}\|_{1}$. Moreover, we set $\mathbf{V}_{\mathbf{0}}:=\left\{\mathbf{v} \in \mathbf{V}:\left.\mathbf{v}\right|_{\Gamma_{D}}=\mathbf{0}\right\}$ and $\mathbf{V}_{D}:=\left\{\mathbf{v} \in \mathbf{V}:\left.\mathbf{v}\right|_{\Gamma_{D}}=\mathbf{u}_{D}\right\}$. For $\mathbf{u}, \mathbf{v} \in \mathbf{V}$, we define the bilinear form $a(\mathbf{u}, \mathbf{v})$ and the linear form $f(\mathbf{v})$ by

$$
a(\mathbf{u}, \mathbf{v}):=\int_{\Omega} \boldsymbol{\sigma}(\mathbf{u}): \varepsilon(\mathbf{v}) \mathrm{d} x, \quad f(\mathbf{v}):=\int_{\Omega} \mathbf{f} \cdot \mathbf{v} \mathrm{d} x .
$$

Let the convex set $\mathbf{K}$ be given by $\mathbf{K}:=\left\{\mathbf{v} \in \mathbf{V}_{D}: \mathbf{v}_{n} \leq 0\right.$ on $\left.\Gamma_{C}\right\}$. Then the solution $\mathbf{u} \in \mathbf{K}$ of the contact problem satisfies the variational inequality

$$
a(\mathbf{u}, \mathbf{v}-\mathbf{u}) \geq f(\mathbf{v}-\mathbf{u}), \quad \mathbf{v} \in \mathbf{K} .
$$

Although our discretization is based on a pure displacement approach, the analysis and the definition of the error estimator use a mixed formulation. Introducing the surface traction on the contact boundary $\Gamma_{C}$ as an additional unknown, the variational inequality (2.4) can be rewritten as a saddle point problem. The Lagrange multiplier space $\mathbf{M}$ is set to be the dual space of the trace space $\mathbf{W}$ of $\mathbf{V}_{\mathbf{0}}$ restricted to $\Gamma_{C}$. By assumption, $\bar{\Gamma}_{C}$ is a compact subset of $\partial \Omega \backslash \bar{\Gamma}_{D}$, and thus we have $\mathbf{W}=\left[H^{\frac{1}{2}}\left(\Gamma_{C}\right)\right]^{2}$. We now define the convex cone of Lagrange multipliers by

$$
\mathbf{M}^{+}:=\left\{\boldsymbol{\mu} \in \mathbf{M}:\langle\boldsymbol{\mu}, \mathbf{v}\rangle \geq 0, \mathbf{v} \in \mathbf{W}^{+}\right\},
$$

where $\langle\cdot, \cdot\rangle$ denotes the duality pairing between $\mathbf{M}$ and $\mathbf{W}$ on $\Gamma_{C}$, and $\mathbf{W}^{+}:=\{\mathbf{v} \in$ $\left.\mathbf{W}: \mathbf{v}_{n} \geq 0\right\}$. It is easy to see that for $\boldsymbol{\mu} \in \mathbf{M}^{+}$, we have $\boldsymbol{\mu} \cdot \mathbf{t}=0$.

Defining the bilinear form $b(\cdot, \cdot)$ on the product space $\mathbf{V} \times \mathbf{M}$ by

$$
b(\mathbf{v}, \boldsymbol{\mu}):=\langle\boldsymbol{\mu}, \mathbf{v}\rangle, \quad \boldsymbol{\mu} \in \mathbf{M}, \mathbf{v} \in \mathbf{V},
$$

we get the saddle point formulation of a contact problem without friction; see, e.g., 28. Chap. 1.3]. We find $\mathbf{u} \in \mathbf{V}_{D}$ and $\boldsymbol{\lambda} \in \mathbf{M}^{+}$such that

$$
\begin{array}{lll}
a(\mathbf{u}, \mathbf{v})+b(\mathbf{v}, \boldsymbol{\lambda}) & =f(\mathbf{v}), & \mathbf{v} \in \mathbf{V}_{0}, \\
b(\mathbf{u}, \boldsymbol{\mu}-\boldsymbol{\lambda}) & \leq 0, & \boldsymbol{\mu} \in \mathbf{M}^{+} .
\end{array}
$$

The existence and uniqueness of $(\mathbf{u}, \boldsymbol{\lambda}) \in \mathbf{V}_{D} \times \mathbf{M}^{+}$has been stated, e.g., in [28, Theorem 3.11 and Remark 3.10]. Moreover, we find that $\boldsymbol{\lambda}=-\boldsymbol{\sigma}(\mathbf{u}) \mathbf{n}$. Setting $\boldsymbol{\mu}=\mathbf{0}$ and $\boldsymbol{\mu}=2 \boldsymbol{\lambda}$, it is easy to see that $b(\mathbf{u}, \boldsymbol{\lambda})=0$. The second line of (2.5) reflects the linearized nonpenetration condition and guarantees that $\mathbf{u} \in \mathbf{K}$. Restricting 
the first line of (2.5) to $\mathbf{v}-\mathbf{u}$ for $\mathbf{v} \in \mathbf{K}$, we find $\mathbf{v}-\mathbf{u} \in \mathbf{V}_{\mathbf{0}}$ and

$$
a(\mathbf{u}, \mathbf{v}-\mathbf{u})=f(\mathbf{v}-\mathbf{u})-b(\mathbf{v}, \boldsymbol{\lambda}) \geq f(\mathbf{v}-\mathbf{u}),
$$

and thus $\mathbf{u}$ solves (2.4).

Remark 2.1. We note that in the case of no friction it would be sufficient to work with a scalar Lagrange multiplier space. Working with the vectorial space $\mathbf{M}$ has the advantage that the approach can be easily generalized to friction.

2.2. Discretization. In this section, we consider a discrete formulation of the variational inequality (2.4) and the saddle point formulation (2.5). A one-body contact problem can be regarded as a simplified case of a two-body contact problem for which mortar techniques with nodal Lagrange finite elements as Lagrange multipliers have been considered and analyzed, e.g., in [7, 8, 9, 23, 29, 30]. Here, we apply these results and work with a dual Lagrange multiplier space; see, e.g., 33. To approximate $\mathbf{V}$, we use standard conforming finite elements of lowest order. For simplicity of notation, we restrict ourselves to the case of a locally quasi-uniform simplicial triangulation, and we assume that the domain $\Omega$ and the sub-boundaries $\Gamma_{C}, \Gamma_{N}$ and $\Gamma_{D}$ are resolved by the triangulation. The finite element space associated with the shape regular triangulation $\mathcal{T}_{h}$ is denoted by $S_{h}(\Omega)$. For ease of presentation, we assume that $\mathbf{u}_{D}$ is a piecewise affine function and can be exactly represented by an element in $\left[S_{h}(\Omega)\right]^{2}$. We define the discrete space $\mathbf{V}_{h}:=\left[S_{h}(\Omega)\right]^{2} \cap \mathbf{V}_{\mathbf{0}}$ and set $\mathbf{V}_{h ; D}:=\left[S_{h}(\Omega)\right]^{2} \cap \mathbf{V}_{D}$. In the mortar context, the Lagrange multiplier space inherits its one-dimensional mesh from the two-dimensional triangulation on the slave side. Here we only have one body which plays the role of the slave side. The discrete Lagrange multiplier space is defined in terms of continuous piecewise cubic nodal basis functions $\psi_{p}, p \in \mathcal{P}_{h}^{C}$, where $\mathcal{P}_{h}^{C}$ denotes the set of nodes on $\bar{\Gamma}_{C}$, [59]; see Figure 1.

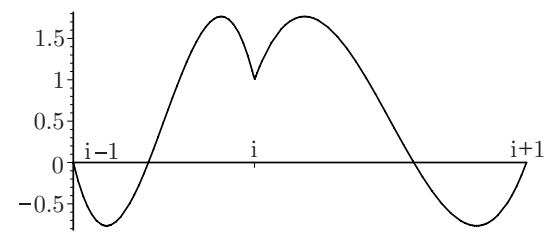

Figure 1. Nodal basis function of a continuous dual Lagrange multiplier space.

We recall that the two sets $\left\{\psi_{p}\right\}_{p}$ and $\left\{\varphi_{p}\right\}_{p}$ form a pair of biorthogonal basis functions, where $\varphi_{p}$ is the nodal Lagrange finite element basis function associated with the node $p$ on the one-dimensional boundary $\bar{\Gamma}_{C}$, i.e., $\int_{\Gamma_{C}} \psi_{p} \varphi_{q} \mathrm{~d} s=$ $\delta_{p q} \int_{\Gamma_{C}} \varphi_{q} \mathrm{~d} s$. The discrete vectorial Lagrange multiplier space is denoted by $\mathbf{M}_{h}$. Let $\mathbf{W}_{h}$ be the vector-valued trace space of $\mathbf{V}_{h}$ restricted to $\Gamma_{C}$. We now define the discrete approximation of $\mathbf{M}^{+}$by

$$
\mathbf{M}_{h}^{+}:=\left\{\boldsymbol{\mu}_{h} \in \mathbf{M}_{h}:\left\langle\boldsymbol{\mu}_{h}, \mathbf{v}_{h}\right\rangle \geq 0, \mathbf{v}_{h} \in \mathbf{W}_{h}^{+}\right\},
$$

where $\mathbf{W}_{h}^{+}:=\left\{\mathbf{v}_{h} \in \mathbf{W}_{h}:\left(\mathbf{v}_{h}\right)_{n} \geq 0\right.$ on $\left.\Gamma_{C}\right\}$. Using the biorthogonality between $\left\{\varphi_{p}\right\}_{p}$ and $\left\{\psi_{p}\right\}$, it is easy to see that each element $\boldsymbol{\mu}_{h} \in \mathbf{M}_{h}^{+}$can be written as $\boldsymbol{\mu}_{h}=\sum_{p \in \mathcal{P}_{h}^{C}} \alpha_{p} \psi_{p} \mathbf{n}, \alpha_{p} \geq 0$. We remark that $\psi_{p} \mathbf{n} \notin \mathbf{M}^{+}$and thus $\mathbf{M}_{h}^{+} \not \subset \mathbf{M}^{+}$. 
With respect to the Lagrange multiplier this approach is a nonconforming finite element technique.

The finite element solution of (2.4) is given by: find $\mathbf{u}_{h} \in \mathbf{K}_{h}$,

$$
a\left(\mathbf{u}_{h}, \mathbf{v}-\mathbf{u}_{h}\right) \geq f\left(\mathbf{v}-\mathbf{u}_{h}\right), \quad \mathbf{v} \in \mathbf{K}_{h},
$$

where $\mathbf{K}_{h}:=\left\{\mathbf{v} \in \mathbf{V}_{h ; D}:\left(\mathbf{v}_{h}\right)_{n} \leq 0\right.$ on $\left.\Gamma_{C}\right\} \subset \mathbf{K}$. In contrast to the Lagrange multiplier, the primal variable $\mathbf{u}_{h}$ can be analyzed within the abstract framework of conforming finite elements. Moreover it is easy to see that the discrete set $\mathbf{K}_{h}$ can be characterized by

$$
\mathbf{K}_{h}=\left\{\mathbf{v} \in \mathbf{V}_{h ; D}: \int_{\Gamma_{C}}\left(\mathbf{v}_{h}\right)_{n} \psi_{p} \mathrm{~d} s \leq 0, p \in \mathcal{P}_{h}^{C}\right\} .
$$

Now, (2.6) can be reformulated as a mixed approach: find $\mathbf{u}_{h} \in \mathbf{V}_{h ; D}$ and $\boldsymbol{\lambda}_{h} \in \mathbf{M}_{h}^{+}$ such that

$$
\begin{aligned}
& a\left(\mathbf{u}_{h}, \mathbf{v}_{h}\right)+b\left(\mathbf{v}_{h}, \boldsymbol{\lambda}_{h}\right)=f\left(\mathbf{v}_{h}\right), \quad \mathbf{v}_{h} \in \mathbf{V}_{h}, \\
& b\left(\mathbf{u}_{h}, \boldsymbol{\mu}_{h}-\boldsymbol{\lambda}_{h}\right) \leq 0, \quad \boldsymbol{\mu}_{h} \in \mathbf{M}_{h}^{+} .
\end{aligned}
$$

Setting $\boldsymbol{\mu}_{h}=\mathbf{0}$ and $\boldsymbol{\mu}_{h}=2 \boldsymbol{\lambda}_{h}$, we obtain the discrete complementary condition $b\left(\mathbf{u}_{h}, \boldsymbol{\lambda}_{h}\right)=0$. We note that if the finite element solution of (2.6) is known, the discrete Lagrange multiplier $\boldsymbol{\lambda}_{h}$ can be locally computed from (2.7) by static condensation. This results from the fact that we work with biorthogonal basis functions, and thus the inverse of a diagonal matrix enters. More precisely, we have $\boldsymbol{\lambda}_{h}=\sum_{p \in \mathcal{P}_{h}^{C}} \beta_{p} \psi_{p} \mathbf{n}$, where $\beta_{p}$ is the local scaled residual given by

$$
\beta_{p}=\frac{f\left(\varphi_{p} \mathbf{n}\right)-a\left(\mathbf{u}_{h}, \varphi_{p} \mathbf{n}\right)}{\int_{\Gamma_{C}} \varphi_{p} \mathrm{~d} s} \geq 0 .
$$

In the case that $\mathbf{M}$ is discretized in terms of nodal Lagrange finite elements, one has to solve a mass matrix system, and the coefficients of the solution vector do not have to be nonnegative.

To show a local upper bound for the error estimator in the contact setting, we use the mortar projection operators $\boldsymbol{\Pi}:\left[H^{\frac{1}{2}}\left(\Gamma_{C}\right)\right]^{2} \rightarrow \mathbf{W}_{h}$ and $\boldsymbol{\Pi}^{*}:\left[H^{-\frac{1}{2}}\left(\Gamma_{C}\right)\right]^{2} \rightarrow$ $\mathbf{M}_{h}$,

$$
\langle\boldsymbol{\mu}, \boldsymbol{\Pi} \mathbf{w}\rangle:=\langle\boldsymbol{\mu}, \mathbf{w}\rangle, \quad \boldsymbol{\mu} \in \mathbf{M}_{h}, \quad\left\langle\boldsymbol{\Pi}^{*} \boldsymbol{\mu}, \mathbf{w}\right\rangle:=\langle\boldsymbol{\mu}, \mathbf{w}\rangle, \quad \mathbf{w} \in \mathbf{W}_{h} .
$$

Then it is easy to see that $\langle\boldsymbol{\mu}, \boldsymbol{\Pi} \mathbf{w}\rangle=\left\langle\boldsymbol{\Pi}^{*} \boldsymbol{\mu}, \mathbf{w}\right\rangle$. It is well known that these operators are stable and satisfy suitable approximation properties, 58, Chapters 1.2.1 and 1.2.2]. We note that due to the biorthogonality of the nodal basis functions of $\mathbf{M}_{h}$ and $\mathbf{W}_{h}$ a local support is preserved under the mappings $\boldsymbol{\Pi}$ and $\boldsymbol{\Pi}^{*}$.

The following local $L^{2}$-stability and properties of $\boldsymbol{\Pi}$ and $\boldsymbol{\Pi}^{*}$ will be used:

$$
\begin{aligned}
\|\mathbf{\Pi} \mathbf{w}\|_{0 ; e} & \leq C\|\mathbf{w}\|_{0 ; s_{e}}, & \mathbf{w} \in\left[L^{2}\left(\Gamma_{C}\right)\right]^{2}, \\
\left\|\boldsymbol{\Pi}^{*} \mathbf{w}\right\|_{0 ; e} & \leq C\|\mathbf{w}\|_{0 ; s_{e}}, & \mathbf{w} \in\left[L^{2}\left(\Gamma_{C}\right)\right]^{2} .
\end{aligned}
$$

Here, $s_{e} \subseteq \Gamma_{C}$ is a local neighborhood of $e$ consisting of at most three edges; see Figure 2. Moreover, we note that from the locality of the support of the basis and the biorthogonality, we can conclude that

$$
\left.\boldsymbol{\Pi} \mathbf{w}\right|_{e}=\left.\boldsymbol{\Pi}^{*} \mathbf{w}\right|_{e}=\left.\mathbf{w}\right|_{e} \quad \text { if } \mathbf{w} \text { is constant on } s_{e} \subseteq \Gamma_{C} .
$$




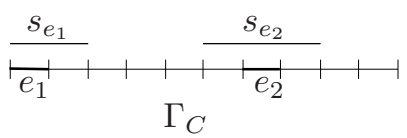

Figure 2. Definition of $s_{e}$.

\section{ERror ESTIMATOR AND A PRIORI ANALYSIS}

Our error estimator is defined in terms of equilibrated fluxes. Equilibrated fluxes are well established and often used for an adaptive error control. We refer to the monographs [2, 6, 55] and the references therein. The basic ideas can be found in the early works [35, 39, and general equilibration techniques for problems in structural mechanics are presented in [40, 48. Equilibrated fluxes in linear elasticity reflect the duality between displacement and traction. From the mathematical point of view, they can be analyzed in the framework of mixed finite elements. Knowing the finite element solution of the displacement-based primal formulation, a consistent flux can be easily computed by a local postprocess. Moreover, the so-computed flux satisfies locally the equilibrium condition and can be used to obtain a symmetric and $H$ (div)conforming approximation for the stress. In contrast to the Laplace operator for which low order $H$ (div)-conforming finite elements for the flux are well known, the construction of symmetric and $H$ (div)-conforming stress approximations is much more involved. There exists no low order finite element; see [4. Therefore, we have to work with a discrete space for the stress which includes locally cubic polynomials. However in the context of a posteriori error estimates, this stress approximation can be easily obtained by a local postprocess from the finite element displacement. Moreover it can provide an upper bound for the error where the constant is one which is of special interest within an adaptive error control. This is not the case if we work with the simpler edge-related approach based on equilibrated fluxes or with a standard residual type estimator. A posteriori error estimates for the displacements in terms of local surface tractions are also considered in [17, 36, 47, 49, 50. Here, we use these ideas and extend the approach to the variational inequality setting.

3.1. Definition of the error estimator. Let us assume from now on that $\boldsymbol{\lambda}_{h}$ is known. Then using the equivalence between (2.6) and (2.7), we find that $\mathbf{u}_{h}$ satisfies a variational equality where $\boldsymbol{\lambda}_{h}$ plays the role of a Neumann boundary condition on $\Gamma_{C}$, i.e.,

$$
a\left(\mathbf{u}_{h}, \mathbf{v}\right)=f(\mathbf{v})-\left\langle\boldsymbol{\lambda}_{h}, \mathbf{v}\right\rangle, \quad \mathbf{v} \in \mathbf{V}_{h} .
$$

Following the construction in [2, we define locally for each edge $e$ of the triangulation $\mathcal{T}_{h}$ a flux $\mathbf{g}_{e} \in\left[P_{1}(e)\right]^{2}$ such that for all $T \in \mathcal{T}_{h}$ and $\mathbf{v} \in\left[P_{1}(T)\right]^{2}$, the local equilibrium

$$
a_{T}\left(\mathbf{u}_{h}, \mathbf{v}\right)=f_{T}(\mathbf{v})+\sum_{e \subset \partial T} \int_{e}\left(\mathbf{n}_{e} \cdot \mathbf{n}_{T}\right) \mathbf{g}_{e} \cdot \mathbf{v} \mathrm{d} s
$$

is satisfied. Here $a_{T}(\cdot, \cdot)$ and $f_{T}(\cdot)$ denote the elementwise contributions of the bilinear form $a(\cdot, \cdot)$ and the linear form $f(\cdot)$, respectively, and $\mathbf{n}_{e}$ stands for a fixed unit normal on the edge $e$, and $\mathbf{n}_{T}$ is the outer unit normal on $\partial T$. If $e \subset \partial \Omega$, we set $\mathbf{n}_{e}=\mathbf{n}$. Additionally, we set on the homogeneous Neumann boundary part $\Gamma_{N}$ the flux to be zero, i.e., $\mathbf{g}_{e}:=\mathbf{0}$. To define $\mathbf{g}_{e}$ on the contact boundary, we use $\boldsymbol{\lambda}_{h}$ 


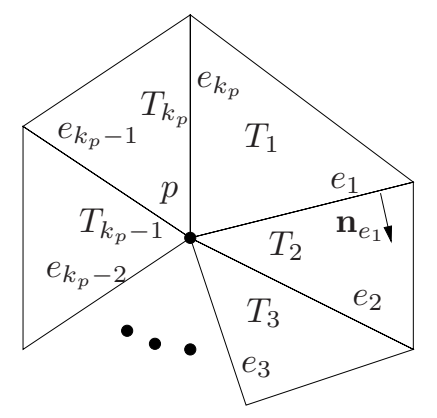

Figure 3. Local numbering of edges and elements.

and set for each edge $e$ on $\Gamma_{C}$,

$$
\int_{e} \mathbf{g}_{e} \cdot \mathbf{v} \mathrm{d} s:=-\int_{e} \boldsymbol{\lambda}_{h} \cdot \mathbf{v} \mathrm{d} s, \quad \mathbf{v} \in\left[P_{1}(e)\right]^{2} .
$$

We note that in our definition, $\Gamma_{C}$ is treated as a Neumann boundary and that the exact surface traction $\boldsymbol{\lambda}$ is replaced by a discrete one, i.e., by $\boldsymbol{\lambda}_{h}$. A unique solution for the equilibrated fluxes can be obtained by solving a minimization problem, i.e., $\mathbf{g}_{e}$ is as close as possible to the average of the discrete flux $\left\{\boldsymbol{\sigma}\left(\mathbf{u}_{h}\right) \mathbf{n}_{e}\right\}$. In order to preserve the locality, we do not minimize $\sum_{e \in \mathcal{E}_{h}}\left\|\mathbf{g}_{e}-\left\{\boldsymbol{\sigma}\left(\mathbf{u}_{h}\right) \mathbf{n}_{e}\right\}\right\|_{0 ; e}^{2}$ but minimize the difference between the moments of the equilibrated flux and the average of the discrete flux. Here $\mathcal{E}_{h}$ denotes the set of all edges in $\mathcal{T}_{h}$. We refer to 2 for details regarding the structure of the linear systems, the minimization step and the different cases of vertices associated with the interior of the domain or boundary conditions.

For the convenience of the reader, we recall the basic steps for an inner node $p$. The moments of $\mathbf{g}_{e}$ are defined by $\mathbf{m}_{e}(p):=\int_{e} \mathbf{g}_{e} \varphi_{p} \mathrm{~d} s$. Using Condition (3.12) for all elements sharing a given vertex $p$ and setting $\mathbf{v}=\varphi_{p} \boldsymbol{e}_{j}$ with the unit vector $\boldsymbol{e}_{j}$, $j \in\{1,2\}$, we see that only the moments $\mathbf{m}_{e}(p)$ for adjacent edges of $p$ enter. By using a clockwise numbering $T_{1}, \ldots, T_{k_{p}}$ for neighboring elements and $e_{1}, \ldots e_{k_{p}}$ for the edges such that $\partial T_{l} \cap \partial T_{l+1}=\bar{e}_{l}$, where $T_{k_{p}+1}:=T_{1}$, see Figure 3. Condition (3.12) reads

$$
\left.\left(\mathbf{n}_{e_{l}} \cdot \mathbf{n}_{T_{l}}\right)\right|_{e_{l}} \mathbf{m}_{e_{l}}(p) \cdot \boldsymbol{e}_{j}+\left.\left(\mathbf{n}_{e_{l+1}} \cdot \mathbf{n}_{T_{l+1}}\right)\right|_{e_{l+1}} \mathbf{m}_{e_{l+1}}(p) \cdot \boldsymbol{e}_{j}=\mathbf{r}_{l+1}(p) \cdot \boldsymbol{e}_{j},
$$

which leads to a $k_{p}$-dimensional linear system for each of the two components of the moments $\mathbf{m}_{e_{l}}(p)$. Here, the size $k_{p}$ is given by the number of triangles sharing the node $p$, and the right-hand side is defined by

$$
\mathbf{r}_{l+1}(p) \cdot \boldsymbol{e}_{j}=a_{T_{l+1}}\left(\mathbf{u}_{h}, \varphi_{p} \boldsymbol{e}_{j}\right)-f_{T_{l+1}}\left(\varphi_{p} \boldsymbol{e}_{j}\right) .
$$

Without loss of generality we can assume that $\mathbf{n}_{e_{l}}=\left.\mathbf{n}_{T_{l}}\right|_{e_{l}}$. Then the associated linear system has the simple form

$$
\left(\begin{array}{ccccc}
\mathbf{I d} & 0 & \cdots & 0 & -\mathbf{I d} \\
-\mathbf{I d} & \mathbf{I d} & \ddots & 0 & 0 \\
0 & -\mathbf{I d} & \mathbf{I d} & \ddots & \vdots \\
\vdots & \ddots & \ddots & \ddots & \vdots \\
0 & 0 & \cdots & -\mathbf{I d} & \mathbf{I d}
\end{array}\right)\left(\begin{array}{c}
\mathbf{m}_{e_{1}}(p) \\
\vdots \\
\mathbf{m}_{e_{k_{p}}}(p)
\end{array}\right)=\left(\begin{array}{c}
\mathbf{r}_{2}(p) \\
\vdots \\
\mathbf{r}_{k_{p}}(p) \\
\mathbf{r}_{1}(p)
\end{array}\right)
$$


where Id is the $2 \times 2$ identity matrix. Obviously the matrix is singular; the kernel has dimension two and is generated by $\left(\boldsymbol{e}_{j}, \ldots, \boldsymbol{e}_{j}\right)^{\top}, j=1,2$. The solvability of the system is guaranteed by the observation that

$$
\sum_{l=1}^{k_{p}} \mathbf{r}_{l}(p) \cdot \boldsymbol{e}_{j}=a\left(\mathbf{u}_{h}, \varphi_{p} \boldsymbol{e}_{j}\right)-f\left(\varphi_{p} \boldsymbol{e}_{j}\right)=0, \quad j=1,2
$$

As already noted, a unique solution is obtained by a local minimization step, i.e., by taking the solution of the linear system (3.14) such that

$$
\sum_{l=1}^{k_{p}}\left\|\mathbf{m}_{e_{l}}(p)-\frac{1}{2}\left|e_{l}\right|\left\{\left.\boldsymbol{\sigma}\left(\mathbf{u}_{h}\right) \cdot \mathbf{n}_{e_{l}}\right|_{e_{l}}\right\}\right\|^{2}
$$

is minimal. For vertices on the boundary the system has to take into account the boundary conditions but similar arguments apply; see [2, 39].

In terms of the equilibrated fluxes, we construct a locally defined but globally $H\left(\right.$ div)-conforming approximation $\boldsymbol{\sigma}_{h}$ of the stress $\boldsymbol{\sigma}(\mathbf{u})$. To do so, we use ArnoldWinther elements; see 4, 57. The stress approximation and error estimator are defined in the same way as in 44 for the Lamé equation. The main difference is in the computation of the equilibrated fluxes on the possible contact zone $\Gamma_{C}$. This step has been worked out in [60] for the more general case of a two-body contact problem. However in [60, the upper and lower bound for the discretization error are not strong enough to guarantee an error decay for the adaptive refinement process. In contrast to the linear Lamé equation, the unknown contact forces have to be replaced by the discrete approximation. For the convenience of the reader, we recall the basic steps and refer to [44, 60, for details.

The Arnold-Winther element is locally defined on $T$ by the 24-dimensional space

$$
X_{T}:=\left\{\boldsymbol{\tau}_{h} \in\left[P_{3}(T)\right]^{2 \times 2}, \quad\left(\boldsymbol{\tau}_{h}\right)_{12}=\left(\boldsymbol{\tau}_{h}\right)_{21}, \quad \operatorname{div} \boldsymbol{\tau}_{h} \in\left[P_{1}(T)\right]^{2}\right\}, \quad T \in \mathcal{T}_{h},
$$

and a global finite element space $X_{h}$ which is $H$ (div)-conforming can be obtained using

- the nodal values (3 dof) at each node $p$,

- the zero and first order moments of $\boldsymbol{\tau}_{h} \mathbf{n}_{e}$ (4 dof) on each edge $e$,

- the mean value (3 dof) on each element $T$

as degrees of freedom; see [4. We note that the subset of $\Gamma_{C}$ where the body is actually in contact with the rigid obstacle is not known a priori. Thus the contact problem can be regarded as a free boundary value problem. In the following, we assume that the actual discrete contact set is a compact subset of $\Gamma_{C}$ in the sense that $\boldsymbol{\lambda}_{h}=\sum_{p \in \mathcal{P}_{h}^{C}} \boldsymbol{\beta}_{p} \psi_{p}$ and $\boldsymbol{\beta}_{p}=\mathbf{0}$ if $p \in \partial \Gamma_{C}$.

We define our stress approximation $\boldsymbol{\sigma}_{h}$ of $\boldsymbol{\sigma}(\mathbf{u})$ by setting

$$
\begin{array}{lll}
\boldsymbol{\sigma}_{h}(p):=\frac{1}{N_{T}^{p}} \sum_{T \in \mathcal{T}_{p}} \boldsymbol{\sigma}\left(\mathbf{u}_{h}\right)_{\mid T}(p)+\boldsymbol{\alpha}(p), & \\
\int_{e} \boldsymbol{\sigma}_{h} \mathbf{n}_{e} \cdot \mathbf{q} \mathrm{d} s:=\int_{e} \mathbf{g}_{e} \cdot \mathbf{q} \mathrm{d} s, & \mathbf{q} \in\left[P_{1}(e)\right]^{2}, \\
\int_{T} \boldsymbol{\sigma}_{h}: \nabla \mathbf{v} \mathrm{d} x:=a_{T}\left(\mathbf{u}_{h}, \mathbf{v}\right), & \mathbf{v} \in\left[P_{1}(T)\right]^{2} .
\end{array}
$$

Here, $\mathcal{T}_{p}$ denotes the set of all triangles having the node $p$ as vertex, and $N_{T}^{p}$ is the number of those triangles. For each node $p$ not on $\partial \Omega \backslash \Gamma_{D}, \boldsymbol{\alpha}(p)=\mathbf{0}$. Otherwise, it 
is defined such that $\boldsymbol{\alpha}(p)$ is a symmetric $2 \times 2$ matrix, $\boldsymbol{\alpha}_{11}(p)^{2}+2 \boldsymbol{\alpha}_{12}(p)^{2}+\boldsymbol{\alpha}_{22}(p)^{2}$ is minimal and $\boldsymbol{\sigma}_{h}(p) \mathbf{n}=\mathbf{0}$ for each node $p$ on $\bar{\Gamma}_{N}$ and $\boldsymbol{\sigma}_{h}(p) \mathbf{n}=-\boldsymbol{\lambda}_{h}(p)=-\boldsymbol{\beta}_{p}$ for each node $p$ on $\Gamma_{C}$. If $p$ is a corner point of the boundary, then $\mathbf{n}$ is not well defined, and we understand the notation $\boldsymbol{\sigma}_{h}(p) \mathbf{n}=\mathbf{0}$ such that $\boldsymbol{\sigma}_{h}(p)=\mathbf{0}$. We note that due to the continuity of the elements in $\mathbf{M}_{h}, \boldsymbol{\alpha}(p)$ is well defined. Moreover, due to the symmetry of $\sigma_{h}$, (3.17) yields only three independent conditions on each element.

Observing that the normal component of the discrete Lagrange multiplier does not have to be positive on $\Gamma_{C}$, we cannot conclude that $b\left(\mathbf{u}, \boldsymbol{\lambda}_{h}\right) \leq 0$. To obtain suitable upper bounds for the error, we introduce an operator $\mathbf{P}_{\mathbf{u}_{h}}$ which depends on the finite element solution $\mathbf{u}_{h}$. This operator maps the normal component of $\boldsymbol{\lambda}_{h}$ on a nonnegative function. A first step towards the definition of $\mathbf{P}_{\mathbf{u}_{h}}$ is the introduction of a mesh-dependent enlarged support of $\mathbf{u}_{h}$ by

$$
\operatorname{suppu}_{h} \subset \operatorname{supp}_{h} \mathbf{u}_{h}:=\bigcup_{\bar{e} \cap \operatorname{supp}_{h} \neq \emptyset} \bar{e},
$$

where we understand the notation $\operatorname{supp}_{h}$ as the support of $\left(\mathbf{u}_{h}\right)_{n}$. The left picture of Figure 4 shows the support of $\mathbf{u}_{h}$, whereas $\operatorname{supp}_{h} \mathbf{u}_{h}$ is depicted in the right picture.
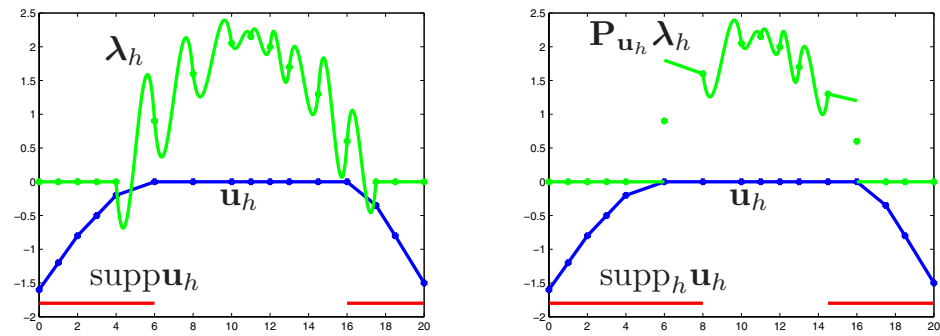

FiguRE 4. Orthogonality between the normal components of $\mathbf{u}_{h}$ and $\boldsymbol{\lambda}_{h}$ (left) and $\mathbf{P}_{\mathbf{u}_{h}} \boldsymbol{\lambda}_{h}$ (right).

Now, we decompose the set $\mathcal{E}_{h}^{C}$ of boundary edges $e \subset \Gamma_{C}$ into three disjoint subsets. $\mathcal{E}_{h}^{s}$ stands for the set of all edges $e \subset \operatorname{suppu}_{h} ; \mathcal{E}_{h}^{i}$ is defined as the set of all edges $e \in \mathcal{E}_{h}^{C}$ such that $e \subset \Gamma_{C} \backslash \operatorname{supp}_{h} \mathbf{u}_{h}$ and $\mathcal{E}_{h}^{b}:=\mathcal{E}_{h}^{C} \backslash\left(\mathcal{E}_{h}^{s} \cup \mathcal{E}_{h}^{i}\right)$. It is easy to see that the elements $e \in \mathcal{E}_{h}^{b}$ can also be characterized by $e \subset \operatorname{supp}_{h} \mathbf{u}_{h} \backslash \operatorname{suppu}_{h}$. For each $e \in \mathcal{E}_{h}^{b}$, we define a neighborhood $\gamma_{e}$ containing at most three elements by

$$
\bar{\gamma}_{e}:=\bar{e} \cup \bigcup_{e_{s} \in \mathcal{E}_{h}^{s} ; \bar{e}_{s} \cap \bar{e} \neq \emptyset} \bar{e}_{s} .
$$

The observation that the support of $\mathbf{u}_{h}$ cannot contain an isolated edge and thus $\gamma_{e} \cap \gamma_{\hat{e}}=\emptyset$ for $e \neq \hat{e}$ yields that for each $e \in \mathcal{E}_{h}^{i} \cup \mathcal{E}_{h}^{s}$,

$$
\gamma_{e}:= \begin{cases}\gamma_{\hat{e}} & \exists \hat{e} \in \mathcal{E}_{h}^{b}: e \subset \gamma_{\hat{e}}, \\ e & \text { else }\end{cases}
$$

is well defined. We note that for $e \in \mathcal{E}_{h}^{i}$, we always get $\gamma_{e}=e$. 
The idea is now to construct the operator $\mathbf{P}_{\mathbf{u}_{h}}$ such that the mean value on each $\gamma_{e}$ is locally preserved, $\mathbf{P}_{\mathbf{u}_{h}} \boldsymbol{\lambda}_{h}$ is zero on $\operatorname{supp} \mathbf{u}_{h}$ and $\mathbf{P}_{\mathbf{u}_{h}} \boldsymbol{\lambda}_{h} \geq 0$. Therefore we introduce the following assumption.

Assumption 3.1. For each edge e $\subset$ supp $\boldsymbol{\lambda}_{h} \cap$ supp $\mathbf{u}_{h}$, we assume that there exists an adjacent edge $\hat{e}$ such that $\hat{e} \subset \Gamma_{C} \backslash \operatorname{supp} \mathbf{u}_{h}$.

This assumption is equivalent to for each edge $e \subset \operatorname{supp} \boldsymbol{\lambda}_{h} \cap \operatorname{supp} \mathbf{u}_{h}$, there exists an $\hat{e} \in \mathcal{E}_{h}^{b}$ such that $e \subset \gamma_{\hat{e}}$ and hence $\gamma_{e}=\gamma_{\hat{e}}$.

The definition of $\mathbf{M}_{h}^{+}$yields that on each edge $e \in \mathcal{E}_{h}^{C}$, we can write $\boldsymbol{\mu}_{h} \in \mathbf{M}_{h}^{+}$as $\boldsymbol{\mu}_{h}=\left(\alpha_{e}^{1} \psi_{e}^{1}+\alpha_{e}^{2} \psi_{e}^{2}\right) \mathbf{n}$, where $\alpha_{e}^{1}, \alpha_{e}^{2} \geq 0$, and $\psi_{e}^{1}, \psi_{e}^{2}$ are the local nodal dual basis functions. In terms of these preliminary observations, we define $\mathbf{P}_{\mathbf{u}_{h}} \boldsymbol{\mu}_{h}, \boldsymbol{\mu}_{h} \in \mathbf{M}_{h}^{+}$, locally on each edge $e \in \mathcal{E}_{h}^{C}$ by

$$
\mathbf{P}_{\mathbf{u}_{h}} \boldsymbol{\mu}_{h}:= \begin{cases}\mathbf{0} & e \in \mathcal{E}_{h}^{s}, \\ \boldsymbol{\mu}_{h} & e \in \mathcal{E}_{h}^{i} \text { and if }\left(\boldsymbol{\mu}_{h}\right)_{n} \geq 0 \text { on } e \\ \left(\alpha_{e}^{1} \varphi_{e}^{1}+\alpha_{e}^{2} \varphi_{e}^{2}\right) \mathbf{n} & e \in \mathcal{E}_{h}^{i} \text { and otherwise } \\ \left(\tilde{\alpha}_{e}^{1} \varphi_{e}^{1}+\tilde{\alpha}_{e}^{2} \varphi_{e}^{2}\right) \mathbf{n} & e \in \mathcal{E}_{h}^{b}\end{cases}
$$

where $\varphi_{e}^{1}, \varphi_{e}^{2}$ are the local nodal Lagrange basis functions associated with the edge $e$ and $\tilde{\alpha}_{e}^{i}:=\alpha_{e}^{i} w_{e}^{i}$, where $w_{e}^{i}:=\int_{\Gamma_{C}} \varphi_{p_{e}^{i}} \mathrm{~d} s / \int_{e} \varphi_{e}^{i} \mathrm{~d} s$ if $\operatorname{supp} \varphi_{p_{e}^{i}} \subset \operatorname{supp}_{h} \mathbf{u}_{h}$ and $w_{e}^{i}:=1$ otherwise. Here $\varphi_{p_{e}^{i}}$ stands for the global basis function associated with the node $p_{e}^{i}$, which can be identified with the local node $i$ of the edge $e$. We remark that in the case of a uniform mesh, $w_{e}^{i} \in\{1,2\}$. As can be easily verified, we obtain $\left(\mathbf{P}_{\mathbf{u}_{h}} \boldsymbol{\mu}_{h}\right)_{n} \geq 0$ and meas $\left(\operatorname{supp} \mathbf{u}_{h} \cap \operatorname{supp} \mathbf{P}_{\mathbf{u}_{h}} \boldsymbol{\mu}_{h}\right)=0$.

Lemma 3.2. Under Assumption 3.1, for each $e \in \mathcal{E}_{h}^{C}$, it follows that

$$
\int_{\gamma_{e}} \mathbf{P}_{\mathbf{u}_{h}} \boldsymbol{\lambda}_{h} \mathrm{~d} s=\int_{\gamma_{e}} \boldsymbol{\lambda}_{h} \mathrm{~d} s .
$$

Proof. For $e \in \mathcal{E}_{h}^{i}$, we have $\gamma_{e}=e$. Then, 3.21 results directly from the definition of $\mathbf{P}_{\mathbf{u}_{h}}$ and the fact that the dual basis functions $\psi_{p}$ and the standard basis functions $\varphi_{p}$ have the same mean value on each edge and that $\boldsymbol{\lambda}_{h} \cdot \mathbf{t}=0$. Let us now consider the case $e \in \mathcal{E}_{h}^{s}$. For $e \notin \operatorname{supp} \boldsymbol{\lambda}_{h}$, we have $\left.\mathbf{P}_{\mathbf{u}_{h}} \boldsymbol{\lambda}_{h}\right|_{e}=\mathbf{0}=\left.\boldsymbol{\lambda}_{h}\right|_{e}$. If $e \in \operatorname{supp} \boldsymbol{\lambda}_{h}$, Assumption 3.1 guarantees the existence of $\hat{e} \in \mathcal{E}_{h}^{b}$ such that $\gamma_{e}=\gamma_{\hat{e}}$. Therefore it remains to prove the case $e \in \mathcal{E}_{h}^{b}$. Here, $\bar{\gamma}_{e}=\bar{e} \cup \bar{e}_{1} \cup \bar{e}_{2}$, where $e_{j}=\emptyset$ or $e_{j}=\hat{e}$ for a suitable $\hat{e} \in \mathcal{E}_{h}^{s}$. By definition of $\mathcal{E}_{h}^{b}$ we find that $e_{j} \subset \operatorname{supp} \boldsymbol{\lambda}_{h} \cap \operatorname{supp} \mathbf{u}_{h}$ and therefore $\alpha_{e_{j}}^{1}=0, \alpha_{e_{j}}^{2}=\alpha_{e}^{j}$ or $\alpha_{e_{j}}^{2}=0, \alpha_{e_{j}}^{1}=\alpha_{e}^{j}$ for $e_{j} \neq \emptyset$. Hence, we have

$$
\int_{e_{j}} \boldsymbol{\lambda}_{h} \mathrm{~d} s=\left(\alpha_{e}^{j} \int_{e_{j}} \psi_{p_{e}^{j}} \mathrm{~d} s\right) \mathbf{n} .
$$


Moreover, by definition, we have $\left.\mathbf{P}_{\mathbf{u}_{h}} \boldsymbol{\lambda}_{h}\right|_{e_{j}}=\mathbf{0}$. A straightforward computation now gives

$$
\begin{aligned}
\int_{\gamma_{e}} \mathbf{P}_{\mathbf{u}_{h}} \boldsymbol{\lambda}_{h} \mathrm{~d} s=\int_{e} \mathbf{P}_{\mathbf{u}_{h}} \boldsymbol{\lambda}_{h} \mathrm{~d} s & =\left(\alpha_{e}^{1} w_{e}^{1} \int_{e} \varphi_{e}^{1} \mathrm{~d} s+\alpha_{e}^{2} w_{e}^{2} \int_{e} \varphi_{e}^{2} \mathrm{~d} s\right) \mathbf{n} \\
& =\left(\alpha_{e}^{1} \int_{e \cup e_{1}} \varphi_{p_{e}^{1}} \mathrm{~d} s+\alpha_{e}^{2} \int_{e \cup e_{2}} \varphi_{p_{e}^{2}} \mathrm{~d} s\right) \mathbf{n} \\
& =\left(\alpha_{e}^{1} \int_{e \cup e_{1}} \psi_{p_{e}^{1}} \mathrm{~d} s+\alpha_{e}^{2} \int_{e \cup e_{2}} \psi_{p_{e}^{2}} \mathrm{~d} s\right) \mathbf{n} \\
& =\int_{\gamma_{e}} \boldsymbol{\lambda}_{h} \mathrm{~d} s .
\end{aligned}
$$

Remark 3.3. Assumption 3.1 excludes isolated nodes $p$ with $\mathbf{u}_{h}(p)=\mathbf{0}$ and $\boldsymbol{\lambda}_{h}(p) \neq$ 0. In this case, $\mathbf{P}_{\mathbf{u}_{h}} \boldsymbol{\lambda}_{h}=\mathbf{0}$ on both neighboring edges of $p$ as both edges are elements of the set $\mathcal{E}_{h}^{s}$, and thus we cannot satisfy (3.21) and $\mathbf{P}_{\mathbf{u}_{h}} \boldsymbol{\lambda}_{h} \cdot \mathbf{u}_{h}=0$ at the same time. These situations can easily occur for coarse meshes but not asymptotically. Such a situation for $h \rightarrow 0$ corresponds to a Lagrange multiplier which is represented by a Delta distribution which is not in $\mathbf{M}$.

Now we are in the situation to define the components of our error estimator. As is quite standard, the global error estimator for the energy norm $\|\mathbf{v}\|_{a}^{2}:=a(\mathbf{v}, \mathbf{v})$ of the error in the displacement is given in terms of local contributions,

$$
\eta^{2}:=\sum_{T \in \mathcal{T}_{h}} \eta_{T}^{2}, \quad \eta_{T}^{2}:=\left\|\mathcal{C}^{-1 / 2}\left(\boldsymbol{\sigma}_{h}-\boldsymbol{\sigma}\left(\mathbf{u}_{h}\right)\right)\right\|_{0 ; T}^{2} .
$$

In addition to $\eta$, we define the quantity

$$
\eta_{C}^{2}:=\sum_{e \in \mathcal{E}_{h}^{C}} \eta_{e}^{2}, \quad \eta_{e}^{2}:=\frac{h_{e}}{\sqrt{2 \mu}}\left\|\boldsymbol{\lambda}_{h}-\mathbf{P}_{\mathbf{u}_{h}} \boldsymbol{\lambda}_{h}\right\|_{0 ; e}^{2} .
$$

3.2. Upper bound for the discretization error. In this subsection, we show that the error estimator yields an upper bound for the discretization error. A preliminary step is to consider the divergence of the stress approximation. We recall a result which has been shown for the more general case of a two-body contact problem in [60 and note that the proof also applies for our simpler situation of a one-body contact problem.

Lemma 3.4. i) Let $\boldsymbol{\sigma}_{h} \in X_{h}$ be defined such that (3.16) and (3.17) hold. Then,

$$
\operatorname{div} \boldsymbol{\sigma}_{h}=-\boldsymbol{\Pi}_{1} \boldsymbol{f},
$$

where $\Pi_{1}$ is the $L^{2}$-projection onto piecewise affine functions.

ii) Let $\boldsymbol{\sigma}_{h} \in X_{h}$ be defined such that (3.15) and (3.16) hold. Then,

$$
\left(\boldsymbol{\sigma}_{h} \mathbf{n}\right)_{\left.\right|_{\Gamma_{N}}}=\mathbf{0} \text { and }\left(\boldsymbol{\sigma}_{h} \mathbf{n}\right)_{\left.\right|_{\Gamma_{C}}}=-\boldsymbol{\lambda}_{h} .
$$

As is standard for a posteriori estimates, we define an oscillation term which only depends on the given data

$$
\xi^{2}:=\sum_{T \in \mathcal{T}_{h}} \xi_{T}^{2}, \quad \xi_{T}^{2}:=\frac{h_{T}^{2}}{2 \mu}\left\|\boldsymbol{f}-\boldsymbol{\Pi}_{1} \boldsymbol{f}\right\|_{0 ; T}^{2} .
$$

In the case of more general boundary conditions, additional terms reflecting the approximation error in the Neumann and Dirichlet boundary conditions enter into 
the definition of $\xi$; see, e.g., 2]. We note that in contrast to [60 no term depending on the unknown solution on $\Gamma_{C}$ enters. Thus we can control $\xi$ within an adaptive refinement process.

Lemma 3.4 results in an upper bound for the discretization error. In a first step, we show that the discretization error can be bounded by $\eta+\eta_{C}$. To estimate the discretization error in terms of the error estimator, the difference $\boldsymbol{\sigma}(\mathbf{u})-\boldsymbol{\sigma}_{h}$ has to be considered.

Theorem 3.5. Under Assumption 3.1, there exist constants $C_{1}, C_{2}<\infty$ independent of the mesh-size such that

$$
\left\|\mathbf{u}-\mathbf{u}_{h}\right\|_{a} \leq \eta+C_{1} \eta_{C}+C_{2} \xi .
$$

Proof. Using the symmetry of Hooke's tensor, we find, for the energy norm of the discretization error in the displacement,

$$
\begin{aligned}
\left\|\mathbf{u}-\mathbf{u}_{h}\right\|_{a}^{2}= & \int_{\Omega}\left(\boldsymbol{\sigma}(\mathbf{u})-\boldsymbol{\sigma}\left(\mathbf{u}_{h}\right)\right):\left(\varepsilon(\mathbf{u})-\varepsilon\left(\mathbf{u}_{h}\right)\right) \mathrm{d} x \\
= & \int_{\Omega}\left(\boldsymbol{\sigma}(\mathbf{u})-\boldsymbol{\sigma}_{h}\right):\left(\varepsilon(\mathbf{u})-\boldsymbol{\varepsilon}\left(\mathbf{u}_{h}\right)\right) \mathrm{d} x \\
& +\int_{\Omega} \mathcal{C}^{-1 / 2}\left(\boldsymbol{\sigma}_{h}-\boldsymbol{\sigma}\left(\mathbf{u}_{h}\right)\right): \mathcal{C}^{1 / 2}\left(\boldsymbol{\varepsilon}(\mathbf{u})-\boldsymbol{\varepsilon}\left(\mathbf{u}_{h}\right)\right) \mathrm{d} x \\
\leq & \eta\left\|\mathbf{u}-\mathbf{u}_{h}\right\|_{a}+\int_{\Omega}\left(\boldsymbol{\sigma}(\mathbf{u})-\boldsymbol{\sigma}_{h}\right):\left(\boldsymbol{\varepsilon}(\mathbf{u})-\boldsymbol{\varepsilon}\left(\mathbf{u}_{h}\right)\right) \mathrm{d} x .
\end{aligned}
$$

Now we have to consider the second term on the right side of (3.24) in more detail. Due to the symmetry and the $H\left(\right.$ div)-conformity of $\boldsymbol{\sigma}_{h}$ and $\boldsymbol{\sigma}(\mathbf{u})$, we can apply integration by parts. Then, the homogeneous Neumann boundary condition on $\Gamma_{N}$, the fact that $\mathbf{u}-\mathbf{u}_{h}=\mathbf{0}$ on $\Gamma_{D}$ and Lemma 3.4 yield

$$
\begin{aligned}
& \int_{\Omega}\left(\boldsymbol{\sigma}(\mathbf{u})-\boldsymbol{\sigma}_{h}\right):\left(\varepsilon(\mathbf{u})-\varepsilon\left(\mathbf{u}_{h}\right)\right) \mathrm{d} x=\int_{\Omega}\left(\boldsymbol{\sigma}(\mathbf{u})-\boldsymbol{\sigma}_{h}\right):\left(\nabla\left(\mathbf{u}-\mathbf{u}_{h}\right)\right) \mathrm{d} x \\
& =-\int_{\Omega} \operatorname{div}\left(\boldsymbol{\sigma}(\mathbf{u})-\boldsymbol{\sigma}_{h}\right) \cdot\left(\mathbf{u}-\mathbf{u}_{h}\right) \mathrm{d} x+\int_{\partial \Omega}\left(\boldsymbol{\sigma}(\mathbf{u})-\boldsymbol{\sigma}_{h}\right) \mathbf{n} \cdot\left(\mathbf{u}-\mathbf{u}_{h}\right) \mathrm{d} s \\
& =\int_{\Omega}\left(\boldsymbol{f}-\boldsymbol{\Pi}_{1} \boldsymbol{f}\right) \cdot\left(\mathbf{u}-\mathbf{u}_{h}\right) \mathrm{d} x-b\left(\mathbf{u}-\mathbf{u}_{h}, \boldsymbol{\lambda}-\boldsymbol{\lambda}_{h}\right) \\
& \leq C\left(\sum_{T \in \mathcal{T}_{h}} \frac{h_{T}^{2}}{2 \mu}\left\|\boldsymbol{f}-\boldsymbol{\Pi}_{1} \boldsymbol{f}\right\|_{0 ; T}^{2}\right)^{\frac{1}{2}}\left\|\mathbf{u}-\mathbf{u}_{h}\right\|_{a}-b\left(\mathbf{u}-\mathbf{u}_{h}, \boldsymbol{\lambda}-\boldsymbol{\lambda}_{h}\right) .
\end{aligned}
$$

Using the orthogonalities, $b\left(\mathbf{u}_{h}, \boldsymbol{\lambda}_{h}\right)=0$ and $b(\mathbf{u}, \boldsymbol{\lambda})=0$, the sign properties $\boldsymbol{\lambda} \in$ $\mathbf{M}^{+}, \boldsymbol{\lambda}_{h} \in \mathbf{M}_{h}^{+}$, the conformity $\mathbf{K}_{h} \subset \mathbf{K}$ and meas $\left(\operatorname{supp} \mathbf{u}_{h} \cap \operatorname{supp} \mathbf{P}_{\mathbf{u}_{h}} \boldsymbol{\lambda}_{h}\right)=0$, we find

$$
\begin{aligned}
b\left(\mathbf{u}_{h}-\mathbf{u}, \boldsymbol{\lambda}-\boldsymbol{\lambda}_{h}\right) & =b\left(\mathbf{u}, \boldsymbol{\lambda}_{h}\right)+b\left(\mathbf{u}_{h}, \boldsymbol{\lambda}\right) \leq b\left(\mathbf{u}, \boldsymbol{\lambda}_{h}-\mathbf{P}_{\mathbf{u}_{h}} \boldsymbol{\lambda}_{h}\right)+b\left(\mathbf{u}, \mathbf{P}_{\mathbf{u}_{h}} \boldsymbol{\lambda}_{h}\right) \\
& \leq b\left(\mathbf{u}, \boldsymbol{\lambda}_{h}-\mathbf{P}_{\mathbf{u}_{h}} \boldsymbol{\lambda}_{h}\right)=b\left(\mathbf{u}-\mathbf{u}_{h}, \boldsymbol{\lambda}_{h}-\mathbf{P}_{\mathbf{u}_{h}} \boldsymbol{\lambda}_{h}\right) \\
& \leq \sum_{e \in \mathcal{E}_{h}^{C}}\left\|\mathbf{u}-\mathbf{u}_{h}\right\|_{\frac{1}{2} ; e}\left\|\boldsymbol{\lambda}_{h}-\mathbf{P}_{\mathbf{u}_{h}} \boldsymbol{\lambda}_{h}\right\|_{-\frac{1}{2} ; e} \\
& \leq C\left\|\mathbf{u}-\mathbf{u}_{h}\right\|_{a}\left(\sum_{e \in \mathcal{E}_{h}^{C}} \frac{1}{2 \mu}\left\|\boldsymbol{\lambda}_{h}-\mathbf{P}_{\mathbf{u}_{h}} \boldsymbol{\lambda}_{h}\right\|_{-\frac{1}{2} ; e}^{2}\right)^{\frac{1}{2}} .
\end{aligned}
$$

To bound the last term by $\eta_{C}$, we use that $\int_{\gamma_{e}} \boldsymbol{\lambda}_{h}-\mathbf{P}_{\mathbf{u}_{h}} \boldsymbol{\lambda}_{h} \mathrm{~d} s=0$; see Lemma 3.2 , Hence, we obtain

$$
\left\|\boldsymbol{\lambda}_{h}-\mathbf{P}_{\mathbf{u}_{h}} \boldsymbol{\lambda}_{h}\right\|_{-\frac{1}{2} ; e}^{2} \leq C\left\|\boldsymbol{\lambda}_{h}-\mathbf{P}_{\mathbf{u}_{h}} \boldsymbol{\lambda}_{h}\right\|_{-\frac{1}{2} ; \gamma_{e}}^{2} \leq C h_{e}\left\|\boldsymbol{\lambda}_{h}-\mathbf{P}_{\mathbf{u}_{h}} \boldsymbol{\lambda}_{h}\right\|_{0 ; \gamma_{e}}^{2} .
$$


We note that no constant depending on Korn's inequality or on the shape regularity of the mesh or on the Lamé parameters enters as a factor in front of $\eta$. The special situation of the variational inequality does not enter directly into the definition of $\eta$. It is only reflected by the term $\eta_{C}$. As is shown in 60, $\eta_{C}$ can be bounded by an additional higher order term on the contact boundary involving the unknown solution. This type of term also enters for general nonhomogeneous Neumann boundary conditions. Unfortunately, $\boldsymbol{\lambda}$ is not a given data, and thus we cannot control this oscillation term within our adaptive refinement algorithm. The next theorem provides a new upper bound for the discretization error in terms of $\eta$ and the data oscillation.

Theorem 3.6. Under Assumption 3.1, there exists a constant $C<\infty$ independent of the mesh-size such that

$$
\left\|\mathbf{u}-\mathbf{u}_{h}\right\|_{a} \leq C(\eta+\xi) .
$$

Proof. Using the result of Theorem 3.5, we have to consider $\eta_{C}$ in more detail and bound it by $\eta$. Using Lemma 3.2 and the definitions of $\mathbf{P}_{\mathbf{u}_{h}} \boldsymbol{\lambda}_{h}$ and of $\boldsymbol{\sigma}_{h}$ gives

$$
\left\|\boldsymbol{\lambda}_{h}-\mathbf{P}_{\mathbf{u}_{h}} \boldsymbol{\lambda}_{h}\right\|_{0 ; e} \leq C h_{e}\left|\boldsymbol{\lambda}_{h}\right|_{1 ; \gamma_{e}} \leq C\left\|\boldsymbol{\lambda}_{h}+\boldsymbol{\sigma}\left(\mathbf{u}_{h}\right) \mathbf{n}\right\|_{0 ; \gamma_{e}}=C\left\|\boldsymbol{\sigma}\left(\mathbf{u}_{h}\right) \mathbf{n}-\boldsymbol{\sigma}_{h} \mathbf{n}\right\|_{0 ; \gamma_{e}} .
$$

Let $\mathcal{T}_{e}$ be the subset of elements in $\mathcal{T}_{h}$ such that each $T \in \mathcal{T}_{e}$ contains one edge of $\gamma_{e}$. We note that the number of elements in $\mathcal{T}_{e}$ is bounded by three. Then, we find, in terms of standard scaling arguments and a discrete inverse estimate,

$$
\eta_{e}^{2} \leq C \sum_{T \in \mathcal{T}_{e}} \eta_{T}^{2}
$$

and thus $\eta_{C} \leq C \eta$.

Remark 3.7. The proof of Theorem 3.6 shows that $\eta_{C}$ can be bounded by $\eta$. More precisely

$$
\eta_{C}^{2} \leq C \sum_{e \in \mathcal{E}_{C}^{n}} \sum_{T \in \mathcal{T}_{e}} \eta_{T}^{2} \leq \beta(h)^{2} \eta^{2},
$$

where $\beta(h) \leq C$ and $\mathcal{E}_{h}^{n} \subset \mathcal{E}_{h}^{C}$ such that $\boldsymbol{\lambda}_{h}-\mathbf{P}_{\mathbf{u}_{h}} \boldsymbol{\lambda}_{h}$ restricted to $e$ is not equal to zero. Then Theorem 3.5 yields

$$
\left\|\mathbf{u}-\mathbf{u}_{h}\right\|_{a} \leq\left(1+C_{1} \beta(h)\right) \eta+C_{2} \xi .
$$

The numerical results show that the number of edges in $\mathcal{E}_{h}^{n}$ is bounded by a small number $n_{m}$ independently of $h$. For most examples, we find $n_{m}=4$. We note that if $\beta(h)$ tends asymptotically to zero, then the upper bound $1+C_{1} \beta(h)$ tends to one.

Corollary 3.8. There exists a constant independent of the mesh-size such that

$$
J\left(\mathbf{u}_{h}\right)-J(\mathbf{u}) \leq C\left(\eta^{2}+\xi^{2}\right),
$$

where the energy $J(\mathbf{v})$ is given by $J(\mathbf{v}):=\frac{1}{2} a(\mathbf{v}, \mathbf{v})-f(\mathbf{v})$.

Proof. The proof of Theorem 3.5 shows that we have the stronger estimate

$$
\left\|\mathbf{u}-\mathbf{u}_{h}\right\|_{a}^{2}-b\left(\mathbf{u}_{h}, \boldsymbol{\lambda}\right) \leq\left(\eta+C_{1} \eta_{C}+C_{2} \xi\right)\left\|\mathbf{u}-\mathbf{u}_{h}\right\|_{a} .
$$

A straightforward computation and Theorem 3.5 and Theorem 3.6 yield that

$$
\begin{aligned}
J\left(\mathbf{u}_{h}\right)-J(\mathbf{u}) & =\frac{1}{2}\left\|\mathbf{u}-\mathbf{u}_{h}\right\|_{a}^{2}-b\left(\mathbf{u}_{h}, \boldsymbol{\lambda}\right) \leq\left\|\mathbf{u}-\mathbf{u}_{h}\right\|_{a}^{2}-b\left(\mathbf{u}_{h}, \boldsymbol{\lambda}\right) \\
& \leq\left(\eta+C_{1} \eta_{C}+C_{2} \xi\right)^{2} \leq C\left(\eta^{2}+\xi^{2}\right) .
\end{aligned}
$$


Remark 3.9. The proof of Theorem 3.5 shows that the constants in the upper bound (3.23) depend on the constant in Korn's inequality but do not depend on the Lamé parameters. In particular, in the nearly incompressible limit, i.e., $\lambda \rightarrow \infty$, the constants $C_{1}$ and $C_{2}$ in (3.23) are uniformly bounded. This is not the case for the constant in Theorem 3.6 and Corollary 3.8. Here the continuity constant of Hooke's tensor $\mathcal{C}$ enters.

3.3. Lower bound for the discretization error. In this subsection, we provide a local upper bound for our error estimator. The structure of this subsection follows 60 , where the more general situation of a two-body problem is considered. However only a weaker result, involving additional terms depending on the unknown contact stress $\boldsymbol{\lambda}$ and the displacement on the contact boundary, has been established.

We will bound the local contribution of our error estimator by $h_{T}\left\|\boldsymbol{\Pi}_{1} \boldsymbol{f}\right\|_{0 ; T}$ and $\sqrt{h_{e}}\left\|\left[\boldsymbol{\sigma}\left(\mathbf{u}_{h}\right) \cdot \mathbf{n}_{e}\right]\right\|_{0 ; e}, e \in \mathcal{E}_{h} \backslash \mathcal{E}_{h}^{C}$. These terms are standard in the estimates for residual type error estimators and can be bounded by the local discretization error and by local higher order terms, i.e.,

$$
\begin{aligned}
h_{T}\left\|\boldsymbol{\Pi}_{1} \boldsymbol{f}\right\|_{0 ; T} & \leq C\left(\left\|\mathbf{u}-\mathbf{u}_{h}\right\|_{a ; T}+\xi_{T}\right), \\
\sqrt{h_{e}}\left\|\left[\boldsymbol{\sigma}\left(\mathbf{u}_{h}\right) \cdot \mathbf{n}_{e}\right]\right\|_{0 ; e}, & \leq C\left(\left\|\mathbf{u}-\mathbf{u}_{h}\right\|_{a ; \omega_{e}}+\xi_{\omega_{e}}\right)
\end{aligned}
$$

see, e.g., 55, 56]. Here, $\omega_{e}$ is defined by the two adjacent elements of $e$. Due to the zero values of the element bubble function on $\partial T$ and due to the fact that only interior edges appear, the variational contact inequality in our setting is not seen. We recall that the proof of (3.25) involves cubic element and quadratic edge bubbles. More precisely, it is based on the observation that on a finite dimensional polynomial space the $L^{2}$-norm $\|\cdot\|_{0 ; \omega}$ and the weighted $L^{2}$-norm $\left\|b_{\omega}^{1 / 2} \cdot\right\|_{0 ; \omega}$ are equivalent. Here, $b_{\omega}>0$ on $\omega$ and $b_{\omega}=0$ on $\partial \omega$ is a smooth bubble function such that $\max _{x \in \omega} b_{\omega}(x)=1$ and $\left|b_{\omega}\right|_{1 ; \omega}^{2}=\mathcal{O}\left(\frac{|\omega|}{h_{\omega}^{2}}\right)$. Replacing the norms on the left side of (3.25) by the weighted $L^{2}$-norms, using the variational equation and integration by parts yields the upper bound; see [55, Chapter 1.2] for details. In general, a posteriori error estimates for linear finite elements involve $\boldsymbol{f}-\boldsymbol{\Pi}_{0} \boldsymbol{f}$ and not $\boldsymbol{f}-\boldsymbol{\Pi}_{1} \boldsymbol{f}$. Here, we work with $\boldsymbol{\Pi}_{1}$ instead of $\boldsymbol{\Pi}_{0}$, which is motivated by the fact that the Arnold-Winther elements have a divergence in $\left[P_{1}(T)\right]^{2}$ and not in $\left[P_{0}(T)\right]^{2}$. However, as noted before, the norm equivalence also holds for polynomials in $\left[P_{1}(T)\right]^{2}$. As we restrict ourselves to edges not on the contact boundary, the result from the linear setting of a variational equality also applies to our variational inequality setting.

Following the proof given in 60] for the two-body case, we get

$$
\begin{aligned}
\eta_{T}^{2} \leq & C \sum_{e \in \mathcal{E}_{T}^{f}} h_{e}\left(\left\|\mathbf{g}_{e}-\left\{\boldsymbol{\sigma}\left(\mathbf{u}_{h}\right)\right\} \mathbf{n}_{e}\right\|_{0 ; e}^{2}+\left\|\left[\boldsymbol{\sigma}\left(\mathbf{u}_{h}\right)\right] \mathbf{n}_{e}\right\|_{0 ; e}^{2}\right) \\
& +\sum_{e \in \mathcal{E}_{T}^{C}} h_{e}\left\|\boldsymbol{\lambda}_{h}+\boldsymbol{\sigma}\left(\mathbf{u}_{h}\right) \mathbf{n}\right\|_{0 ; e}^{2},
\end{aligned}
$$

where $\mathcal{E}_{T}^{C}$ is the subset of edges which have a vertex of $T$ as endpoint and which are on the contact boundary $\Gamma_{C}$ and $\mathcal{E}_{T}^{f}:=\mathcal{E}_{T} \backslash \mathcal{E}_{T}^{C}$. Here $\mathcal{E}_{T}$ denotes all edges sharing one vertex with $T$; see Figure 5. The proof of this local upper bound is based on a discrete mesh-dependent norm which is equivalent to the $L^{2}$-norm on the local Arnold-Winther element $X_{T}$. This equivalence has been shown in [4] and 

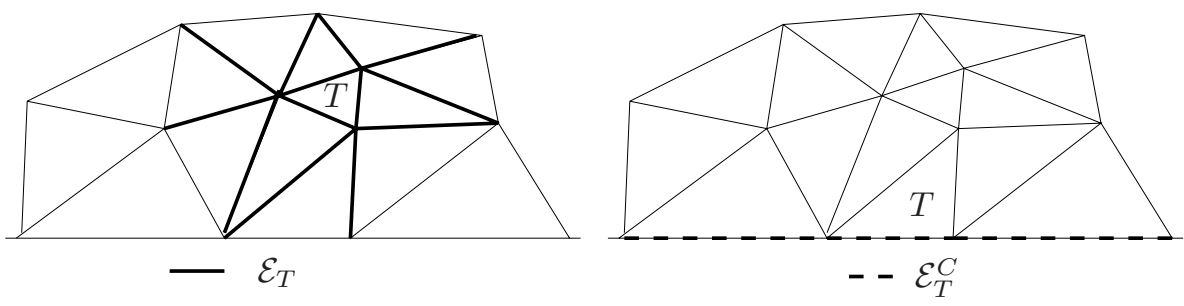

FiguRE 5. Definition of $\mathcal{E}_{T}$ and $\mathcal{E}_{T}^{C}$.

has also been used in [44. The upper bound in (3.26) involves boundary terms on $\Gamma_{C}$ which have to be eliminated. However, the first two terms are standard terms in the definition of a residual-based and an equilibrated flux-based error estimator, see [2, 6, 54, and can be bounded by the element and edge residuals. Because $e \in \mathcal{E}_{T}^{f}$ is an interior edge or an edge on $\overline{\Gamma_{D} \cup \Gamma_{N}}$, both of them are bounded in terms of (3.25). We recall that we have homogeneous Neumann boundary conditions, and thus no additional term reflecting the Neumann data oscillations occurs.

In the following, we have to consider the third term in (3.26) in more detail. To bound $h_{e}\left\|\boldsymbol{\lambda}_{h}+\boldsymbol{\sigma}\left(\mathbf{u}_{h}\right) \mathbf{n}\right\|_{0 ; e}^{2}, e \in \mathcal{E}_{h}^{C}, e \subset \partial T$, we use a decomposition into normal and tangential parts, i.e.,

$$
\left\|\boldsymbol{\lambda}_{h}+\boldsymbol{\sigma}\left(\mathbf{u}_{h}\right) \mathbf{n}\right\|_{0 ; e}^{2}=\left\|\boldsymbol{\lambda}_{h} \cdot \mathbf{n}+\boldsymbol{\sigma}\left(\mathbf{u}_{h}\right) \mathbf{n} \cdot \mathbf{n}\right\|_{0 ; e}^{2}+\left\|\boldsymbol{\lambda}_{h} \cdot \mathbf{t}+\boldsymbol{\sigma}\left(\mathbf{u}_{h}\right) \mathbf{n} \cdot \mathbf{t}\right\|_{0 ; e}^{2} .
$$

Observing that we have a contact problem without friction, we have $\boldsymbol{\lambda}_{h} \cdot \mathbf{t}=0$ and $\boldsymbol{\sigma}(\mathbf{u}) \mathbf{n} \cdot \mathbf{t}=0$ on $\Gamma_{C}$ and thus $\left\|\boldsymbol{\lambda}_{h} \cdot \mathbf{t}+\boldsymbol{\sigma}\left(\mathbf{u}_{h}\right) \mathbf{n} \cdot \mathbf{t}\right\|_{0 ; e}^{2}=\left\|\boldsymbol{\sigma}\left(\mathbf{u}_{h}\right) \mathbf{n} \cdot \mathbf{t}\right\|_{0 ; e}^{2}$. Using the quadratic edge bubble function $b_{e}$, we find by integration by parts

$$
\begin{aligned}
\sqrt{h_{e}}\left\|\boldsymbol{\sigma}\left(\mathbf{u}_{h}\right) \mathbf{n} \cdot \mathbf{t}\right\|_{0 ; e} & =C\left|\int_{e} \boldsymbol{\sigma}\left(\mathbf{u}_{h}\right) \mathbf{n} \cdot\left(\mathbf{t} b_{e}\right) \mathrm{d} s\right|=C\left|\int_{e}\left(\boldsymbol{\sigma}\left(\mathbf{u}_{h}\right)-\boldsymbol{\sigma}(\mathbf{u})\right) \mathbf{n} \cdot\left(\mathbf{t} b_{e}\right) \mathrm{d} s\right| \\
& \leq C\left|\int_{T}\left(\boldsymbol{\sigma}\left(\mathbf{u}_{h}\right)-\boldsymbol{\sigma}(\mathbf{u})\right): \varepsilon\left(\mathbf{t} b_{e}\right) \mathrm{d} x\right|+C\left|\int_{T} \boldsymbol{f} \cdot\left(\mathbf{t} b_{e}\right) \mathrm{d} x\right| \\
& \leq C\left(\left\|\mathbf{u}-\mathbf{u}_{h}\right\|_{a ; T}+\xi_{T}+h_{T}\left\|\boldsymbol{\Pi}_{1} \boldsymbol{f}\right\|_{0 ; T}\right) .
\end{aligned}
$$

For the normal component, we insert the projection $\Pi^{*}$ which is the scalar variant of the one given in (2.8) and get

$$
\left\|\left(\boldsymbol{\lambda}_{h}+\boldsymbol{\sigma}\left(\mathbf{u}_{h}\right) \mathbf{n}\right) \cdot \mathbf{n}\right\|_{0 ; e} \leq\left\|\boldsymbol{\lambda}_{h} \cdot \mathbf{n}+\Pi^{*} \sigma_{n}\right\|_{0 ; e}+\left\|\Pi^{*} \sigma_{n}-\sigma_{n}\right\|_{0 ; e},
$$

where we denote $\boldsymbol{\sigma}\left(\mathbf{u}_{h}\right) \mathbf{n} \cdot \mathbf{n}$ by $\sigma_{n}$. We start with the second term and use that the mortar projection locally reproduces constants; see (2.10).

In terms of the local $L^{2}$-stability (2.9) of $\Pi^{*}$, we find

$$
\left\|\Pi^{*} \sigma_{n}-\sigma_{n}\right\|_{0 ; e}=\left\|\Pi^{*}\left(\sigma_{n}-\sigma_{n, e}\right)-\left(\sigma_{n}-\sigma_{n, e}\right)\right\|_{0 ; e} \leq C\left\|\sigma_{n}-\sigma_{n, e}\right\|_{0 ; s_{e}} .
$$

Here, we denote by $\sigma_{n, e}$ the constant $\left.\boldsymbol{\sigma}\left(\mathbf{u}_{h}\right)\right|_{e} \mathbf{n} \cdot \mathbf{n}$ extended to $\Gamma_{C}$. This difference can be written in terms of the jump between neighboring edges, and we obtain

$$
\left\|\sigma_{n}-\sigma_{n, e}\right\|_{0 ; s_{e}} \leq C \sqrt{h_{e}} \sum_{i=1}^{2}\left\|\left(\boldsymbol{\sigma}\left(\mathbf{u}_{h}\right)_{\left.\right|_{T}}\left(p_{i}^{e}\right)-\boldsymbol{\sigma}\left(\mathbf{u}_{h}\right)_{\left.\right|_{i} ^{e}}\left(p_{i}^{e}\right)\right) \mathbf{n}\right\|,
$$

where $p_{i}^{e}, i=1,2$, denote the two endpoints of the edge $e$ and $T_{i}^{e} \neq T$ is the element sharing the node $p_{i}^{e}$ and having an edge on the contact boundary; see Figure 6 . If such an element does not exist, i.e., if $p_{i}^{e} \in \partial \Gamma_{C}$, we formally set $T_{i}^{e}=T$. The jump 


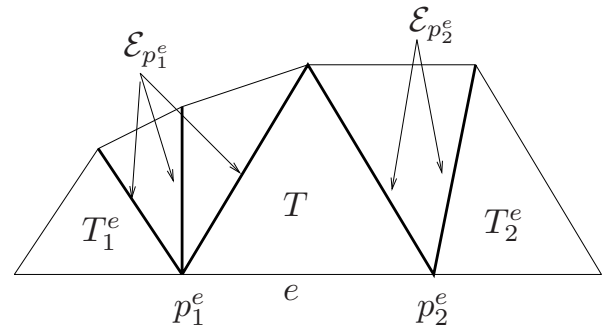

Figure 6. Definition of $p_{i}^{e}, T_{i}^{e}$ and $\mathcal{E}_{p_{i}^{e}}$.

at the nodes can easily be bounded by the jump on the edges

$$
\begin{aligned}
\sqrt{h_{e}}\left\|\left(\boldsymbol{\sigma}\left(\mathbf{u}_{h}\right)_{\left.\right|_{T}}\left(p_{i}^{e}\right)-\boldsymbol{\sigma}\left(\mathbf{u}_{h}\right)_{\left.\right|_{T_{i}^{e}}}\left(p_{i}^{e}\right)\right) \mathbf{n}\right\| & \leq C \sum_{\hat{e} \in \mathcal{E}_{p_{i}^{e}}}\left\|\left[\boldsymbol{\sigma}\left(\mathbf{u}_{h}\right)\right]\right\|_{0 ; \hat{e}} \\
& \leq C \sum_{\hat{e} \in \mathcal{E}_{p_{i}^{e}}}\left\|\left[\boldsymbol{\sigma}\left(\mathbf{u}_{h}\right) \mathbf{n}_{\hat{e}}\right]\right\|_{0 ; \hat{e}},
\end{aligned}
$$

where $\mathcal{E}_{p_{i}^{e}}$ stands for the set of all interior edges sharing the node $p_{i}^{e}$. Here we have used that the jump of $\boldsymbol{\sigma}\left(\mathbf{u}_{h}\right)$ on the edges can be bounded by the jump of $\boldsymbol{\sigma}\left(\mathbf{u}_{h}\right) \mathbf{n}$; see 44 .

To bound the first term on the right side of (3.27), we use that $\boldsymbol{\lambda}_{h} \cdot \mathbf{n}+\Pi^{*} \sigma_{n} \in$ $M_{h}:=\mathbf{M}_{h} \cdot \mathbf{n}$ and can therefore be written on $e$ as $\alpha \psi_{p_{1}^{e}}+\beta \psi_{p_{2}^{e}}$. Setting $v_{h}=$ $\operatorname{sign}(\alpha) \varphi_{p_{1}^{e}}+\operatorname{sign}(\beta) \varphi_{p_{2}^{e}}$, and using the biorthogonality of $\varphi_{p}$ and $\psi_{q}$, a simple calculation gives

$\left\|\alpha \psi_{p_{1}^{e}}+\beta \psi_{p_{2}^{e}}\right\|_{0, e} \leq \frac{C}{\sqrt{h_{e}}} \int_{\Gamma_{C}}\left(\alpha \psi_{p_{1}^{e}}+\beta \psi_{p_{2}^{e}}\right) v_{h} \mathrm{~d} s=\frac{C}{\sqrt{h_{e}}} \int_{\Gamma_{C}}\left(\boldsymbol{\lambda}_{h}+\boldsymbol{\sigma}\left(\mathbf{u}_{h}\right) \mathbf{n}\right) \cdot \mathbf{n} v_{h} \mathrm{~d} s$.

To bound this term, we apply Green's formula on each element and find

$$
\begin{aligned}
\int_{\Gamma_{C}}\left(\boldsymbol{\lambda}_{h}+\boldsymbol{\sigma}\left(\mathbf{u}_{h}\right) \mathbf{n}\right) & \cdot \mathbf{n} v_{h} \mathrm{~d} s=f\left(v_{h} \mathbf{n}\right)-\sum_{i=1}^{2} \sum_{\hat{e} \in \mathcal{E}_{p_{i}^{e}}} \int_{\hat{e}}\left[\boldsymbol{\sigma}\left(\mathbf{u}_{h}\right) \mathbf{n}_{\hat{e}}\right] \cdot v_{h} \mathbf{n} \mathrm{d} s \\
& \leq C\left(\sum_{T \in \mathcal{T}_{e}} h_{T}\left\|\mathbf{\Pi}_{1} \boldsymbol{f}\right\|_{0 ; T}+\sum_{i=1}^{2} \sum_{\hat{e} \in \mathcal{E}_{p_{i}^{e}}} \sqrt{h_{\hat{e}}}\left\|\left[\boldsymbol{\sigma}\left(\mathbf{u}_{h}\right) \mathbf{n}_{\hat{e}}\right]\right\|_{0 ; \hat{e}}\right),
\end{aligned}
$$

where $\mathcal{T}_{e}$ stands for the set of all elements which are in the support of $\varphi_{p_{1}^{e}}$ and $\varphi_{p_{2}^{e}}$, and $\mathcal{E}_{p_{i}^{e}}$ is the set of all interior edges having $p_{i}^{e}$ as endpoint. Here, we have used that $f\left(v_{h} \mathbf{n}\right)=\int_{\Omega} \boldsymbol{\Pi}_{1} \boldsymbol{f} \cdot v_{h} \mathbf{n} \mathrm{d} x$ and that $v_{h} \mathbf{n}$ has a support in $\bigcup_{T \in \mathcal{T}_{e}} T$. Moreover, the $L^{2}$-norms on the elements and edges are bounded by $\left\|v_{h} \mathbf{n}\right\|_{0 ; T} \leq C h_{T}$ and $\left\|v_{h} \mathbf{n}\right\|_{0 ; \hat{e}} \leq C \sqrt{h_{\hat{e}}}$. Finally, by (3.27) and using the local shape regularity of the mesh, we get the upper bound

$$
\begin{aligned}
h_{e} \| \boldsymbol{\lambda}_{h} & +\boldsymbol{\sigma}\left(\mathbf{u}_{h}\right) \mathbf{n} \|_{0 ; e}^{2} \\
\leq & C\left(\sum_{T \in \mathcal{T}_{e}} h_{T}^{2}\left\|\boldsymbol{\Pi}_{1} \boldsymbol{f}\right\|_{0 ; T}^{2}+\sum_{i=1}^{2} \sum_{\hat{e} \in \mathcal{E}_{p_{i}^{e}}} h_{\hat{e}}\left\|\left[\boldsymbol{\sigma}\left(\mathbf{u}_{h}\right) \mathbf{n}_{\hat{e}}\right]\right\|_{0 ; \hat{e}}^{2}\right) .
\end{aligned}
$$

Combining (3.25), (3.26) and (3.28), we get the following lower bound for the discretization error. 
Theorem 3.10. The element contribution $\eta_{T}$ of the error estimator can be bounded by the error on a local neighborhood $\omega_{T}$ and some local oscillation terms

$$
\eta_{T}^{2} \leq C \sum_{\hat{T} \in \mathcal{T}_{h}, \hat{T} \subset \omega_{T}}\left(\left\|\mathbf{u}-\mathbf{u}_{h}\right\|_{a ; \hat{T}}^{2}+\xi_{\hat{T}}^{2}\right) .
$$

The number of elements in $\omega_{T}$ does not depend on $h$ but only on the shape regularity of $\mathcal{T}_{h}$.

\section{NumericAl RESUlts}

In this section, we apply our error estimator to several contact problems and use it to obtain adaptively generated meshes.

4.1. Square on a plane. In our first example, we consider a square that is pushed onto a rigid plane; see Figure 7 . The square is given by $\Omega=(0,1)^{2}$. In addition to the force applied on the top, we compress the square by forces on the left and the right boundary of the square. An additional issue is to prevent corner singularities; hence we use as the boundary force on the noncontact sides $g=-200 p \mathbf{n}$, where $\mathbf{n}$ is the outer normal of the corresponding side and $p=x^{2}(1-x)^{2}$ on the upper side and $p=y^{2}(1-y)^{2}$ on the left and right sides. The material parameters are given by $E=200$ and $\nu=0.3$. This problem setting leads to a nontrivial contact zone as can be seen in the right picture of Figure 7 Here, the contact pressure is shown. In the middle picture, the effective von Mises stress is given.
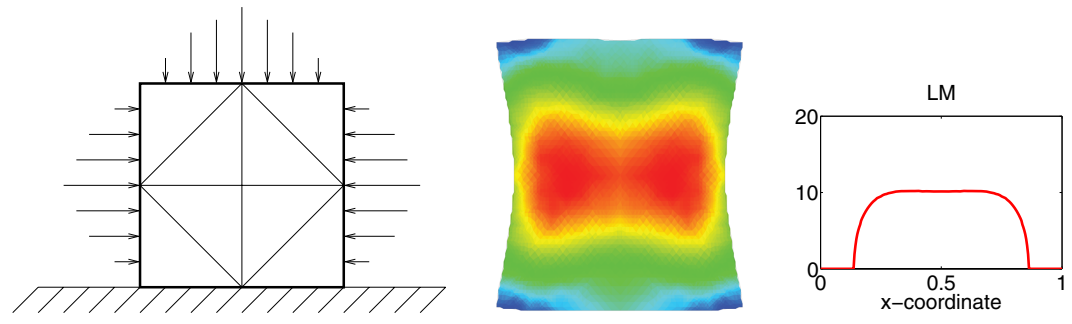

Figure 7. Square on plane: Problem setting with initial mesh, solution and contact stress.
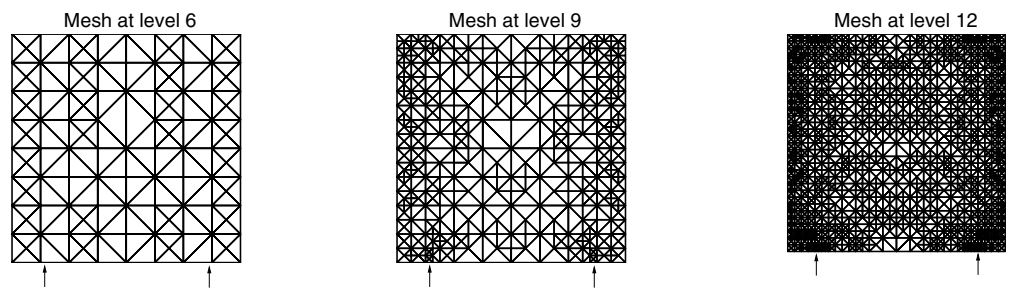

Figure 8. Square on plane: Mesh on level 6, 9 and 12.

For the mesh refinement, we use a bisection strategy based on the error estimator $\eta$. The meshes at different levels are depicted in Figure 8 Here, we see that our adaptive strategy leads at the beginning to an almost uniform mesh refinement which can be explained by the high regularity of the solution of the given contact 
problem. In the middle picture, the mesh on level 9 is shown. We observe at the two endpoints of the contact zone (marked by an arrow) the refinement is higher, whereas in the interior of the actual contact zone the mesh is relatively coarse.

Additionally, we consider the error reduction; see Figure 9. In the left picture, we compare the convergence rates using our adaptive mesh refinement with uniform mesh refinement. Using a uniform mesh refinement, we observe an error of $\mathcal{O}(h)$, which indicates that our problem has $H^{2}$-regularity. In the right picture, we compare the estimated error $\eta$ with the extra term $\eta_{C}$. Here, we define the

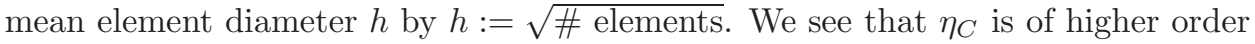
compared to $\eta$ and can thus asymptotically be neglected.
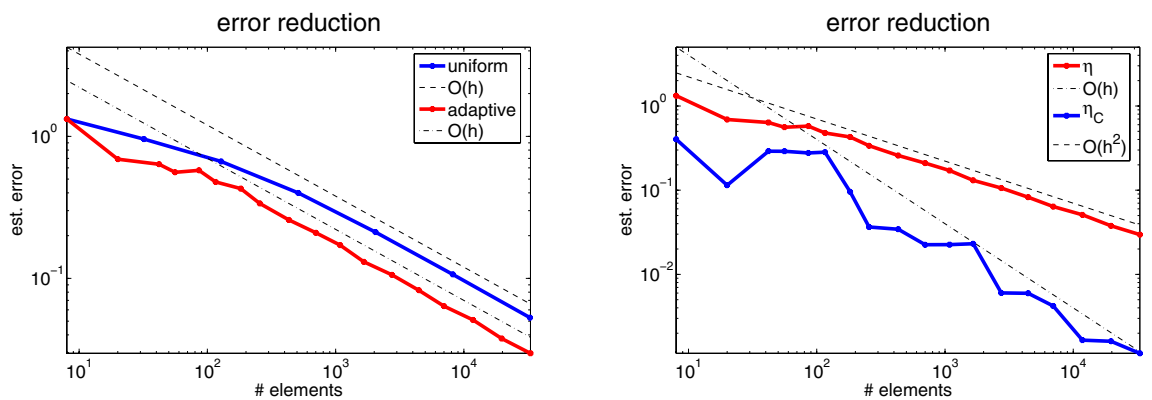

FiguRE 9. Square on plane: Estimated error, left: uniform/adaptive, right: $\eta / \eta_{C}$.

4.2. Square on a circle. In the second example, we push the square of Example 1 on a rigid circle with radius 1 and midpoint $(0.5,-1)$. Here, we apply inhomogeneous Neumann boundary conditions only on the top with $g=50 x(1-x) \mathbf{n}$. As material parameters, we use $E=7000$ and $\nu=0.3$. The problem setting and solution are depicted in Figure 10.
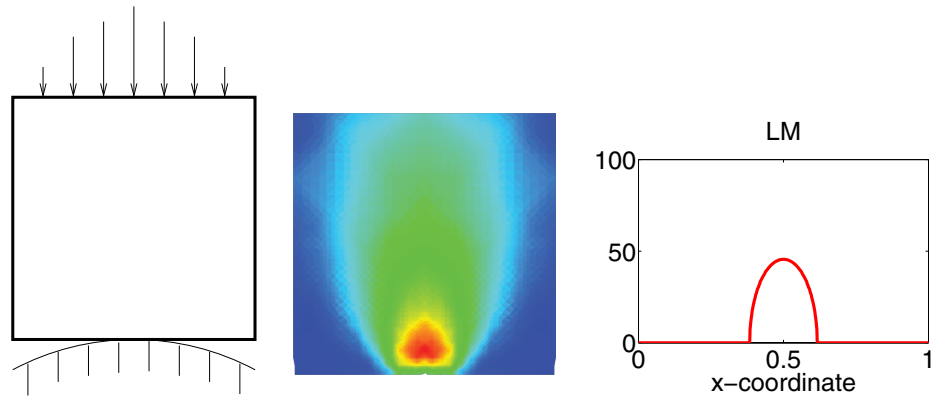

Figure 10. Square on circle: Problem setting, solution and contact stress.

In Figure 11. we show the meshes obtained by bisection using the error estimator $\eta$. Here we see, in comparison to the first example, that the meshes are refined much more locally. As a result, the quantitative error decay obtained by our adaptive algorithm is better than the one obtained by uniform mesh refinement; see Figure 12, As in Example 1, we observe that the ratio $\eta_{C} / \eta$ tends asymptotically to zero. 

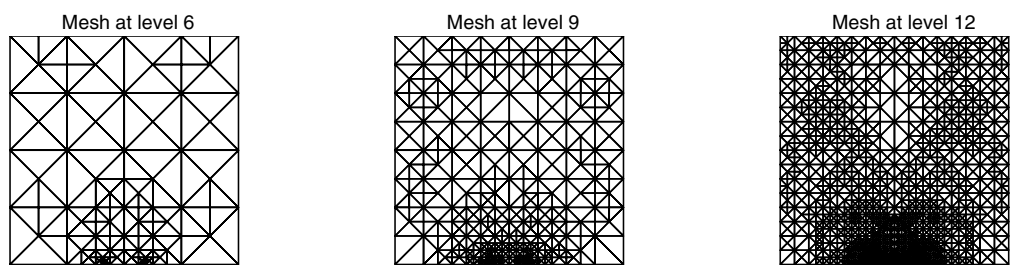

Figure 11. Square on circle: Mesh on levels 6, 9 and 12.
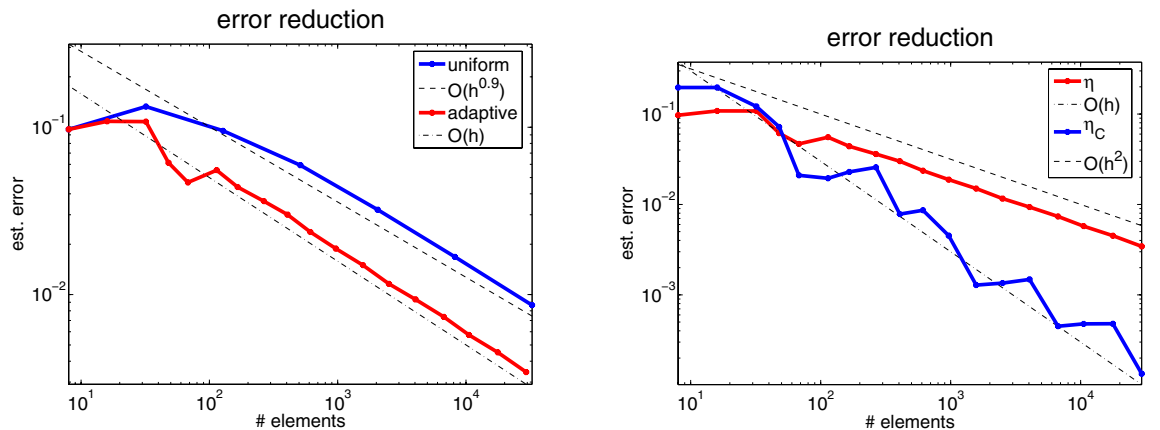

Figure 12. Square on circle: Estimated error, left: uniform/adaptive, right: $\eta / \eta_{C}$.

4.3. Square on a triangle. To get contact problems with low regularity, we push the square onto a triangle with different angles $\alpha$; see Figure 13. Here, the regularity of the solution depends on the angle $\alpha$. In our tests, we use $\alpha=\pi / 6, \alpha=\pi / 4$ and $\alpha=\pi / 3$. The material parameters of the square are given by $E=200, \nu=0.3$. We use the same boundary conditions as in Example 2.
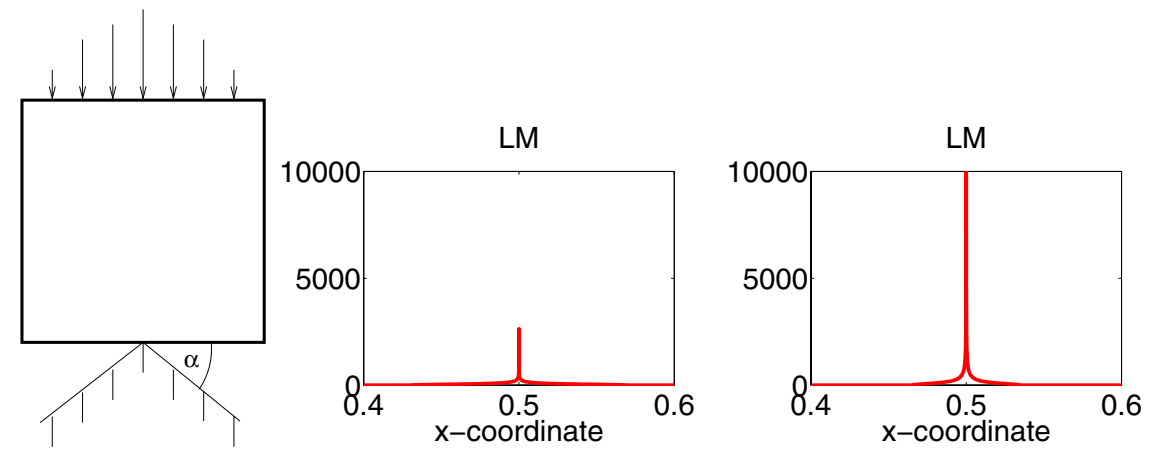

Figure 13. Square on triangle: Problem setting (left) and contact stress $\alpha=\pi / 6$ (middle) and $\alpha=\pi / 3$ (right).

Due to the decreasing regularity for increasing $\alpha$ the adaptive meshes differ for the different values of $\alpha$; see Figure 14. We observe that for $\alpha=\pi / 3$, the mesh is much more locally refined compared to $\alpha=\pi / 6$. 

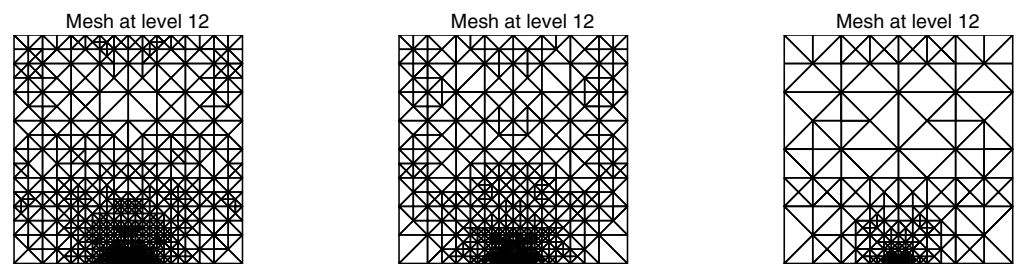

Figure 14. Square on triangle: Mesh on level 12 for $\alpha=\pi / 6, \pi / 4, \pi / 3$.

The regularity can be estimated by observing the convergence rates for the uniform refinement; see Figure 15. Here, we get a convergence rate of approximately $\mathcal{O}\left(h^{0.7}\right)$ for $\alpha=\pi / 6, \mathcal{O}\left(h^{0.5}\right)$ for $\alpha=\pi / 4$ and $\mathcal{O}\left(h^{0.3}\right)$ for $\alpha=\pi / 3$. However, using adaptive mesh refinement, we observe $\mathcal{O}(h)$ in all cases.
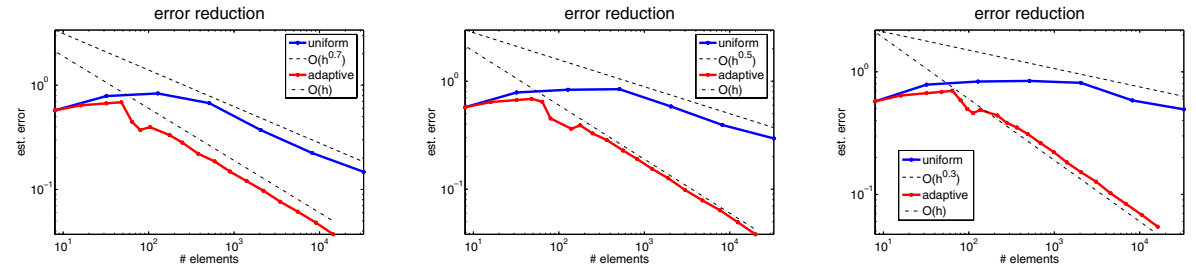

Figure 15. Square on triangle: Estimated error (adaptive/uniform) for $\alpha=\pi / 6, \pi / 4, \pi / 3$.

Again we see that the error contribution $\eta_{C}$ decreases much faster compared to $\eta$; see Figure 16. However, for low regularities, we do not observe that $\eta_{C}$ is of order $\mathcal{O}\left(h^{2}\right)$. To get a better understanding of the situation, we define

$$
\eta_{C}^{\text {contact }}:=\left(\sum_{e \in \mathcal{E}_{h}^{C} \backslash \mathcal{E}_{h}^{i}} \eta_{e}^{2}\right)^{1 / 2} \leq \eta_{C} .
$$
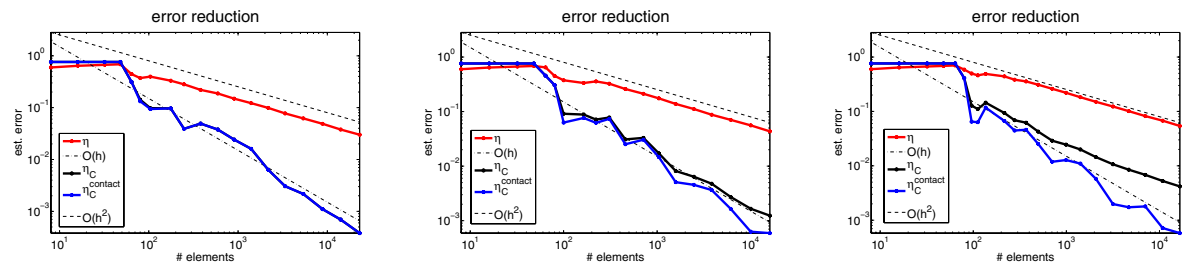

Figure 16. Square on triangle: Comparison of $\eta, \eta_{C}$ and $\eta_{C}^{\text {contact }}$ for $\alpha=\pi / 6, \pi / 4, \pi / 3$.

If the singularity in $\boldsymbol{\lambda}$ is small enough, we find asymptotically $\eta_{C}^{\text {contact }}=\eta_{C}$ and that $\eta_{C}$ is of order $\mathcal{O}\left(h^{2}\right)$. However, for strong singularities in $\boldsymbol{\lambda}$, there are nontrivial contributions $\eta_{e}, e \in \mathcal{E}_{h}^{i}$. If $e \in \mathcal{E}_{h}^{i} \cap \mathcal{E}_{h}^{n}$ is in the actual contact area of the solution $\mathbf{u}$, then $\int_{e} \mathbf{u} \cdot\left(\boldsymbol{\lambda}_{h}-\mathbf{P}_{\mathbf{u}_{h}} \boldsymbol{\lambda}_{h}\right) \mathrm{d} s=0$ although $\boldsymbol{\lambda}_{h} \neq \mathbf{P}_{\mathbf{u}_{h}} \boldsymbol{\lambda}_{h}$. This is the case in our situation; thus the error is bounded by $\eta+C_{1} \eta_{C}^{\text {contact }}+C_{2} \xi$, and $\eta_{C}^{\text {contact }} / \eta$ tends to zero for all three values of $\alpha$. 


\section{UPPER BOUND FOR THE ERROR IN THE LAGRANGE MULTIPLIER}

In this section, we show that the error in the Lagrange multiplier can be bounded in terms of the discretization error in the displacement and additional data oscillation terms. The abstract framework of mixed finite elements in combination with a discrete uniform inf-sup condition yields the standard a priori estimate

$$
\left\|\boldsymbol{\lambda}-\boldsymbol{\lambda}_{h}\right\|_{-\frac{1}{2} ; \Gamma_{C}} \leq C\left(\inf _{\boldsymbol{\mu}_{h} \in \mathbf{M}_{h}}\left\|\boldsymbol{\lambda}-\boldsymbol{\mu}_{h}\right\|_{-\frac{1}{2} ; \Gamma_{C}}+\left\|\mathbf{u}-\mathbf{u}_{h}\right\|_{a}\right) .
$$

If the solution is smooth enough, the first term on the right side is $\mathcal{O}\left(h^{3 / 2}\right)$, whereas the second term is in general only of order $h$. Unfortunately, the first term cannot be controlled a priori because the contact stress is not included in the given data.

Let $\mathbf{V}_{0 ; h}:=\mathbf{V}_{h} \cap\left[H_{0}^{1}(\Omega)\right]^{2}$. Then there exists a space $\mathbf{X}_{h}$ such that $\operatorname{dim} \mathbf{X}_{h}=$ $\operatorname{dim} \mathbf{V}_{0 ; h}$ and moreover $\mathbf{X}_{h}=\operatorname{span}\left\{\chi_{p} \boldsymbol{e}_{i}, i=1,2, p \in \mathcal{P}_{h}^{0}\right\}$, where the basis functions $\chi_{p}$ satisfy

- $\int_{\Omega} \chi_{p} \varphi_{q} \mathrm{~d} x=\delta_{p q} \int_{\Omega} \varphi_{q} \mathrm{~d} x, \quad p, q \in \mathcal{P}_{h}^{0}$,

- $\sum_{p \in \mathcal{P}_{h}^{0}} \chi_{p}=1$,

- $\operatorname{supp} \chi_{p}=\operatorname{supp} \varphi_{p}, p \in \tilde{\mathcal{P}}_{h}^{0}$.

Here $\mathcal{P}_{h}^{0}$ stands for the set of all nodes in the interior of $\Omega$ and $\tilde{\mathcal{P}}_{h}^{0} \subset \mathcal{P}_{h}^{0}$ such that $\operatorname{supp} \varphi_{p} \subset \Omega$ for $p \in \tilde{\mathcal{P}}_{h}^{0}$. For $p \in \tilde{\mathcal{P}}_{h}^{0}$, we set $\chi_{p}=3 \varphi_{p}-\sum_{q \in \mathcal{P}_{p}} \varphi_{q}$ on $\operatorname{supp} \varphi_{p}$, where $\mathcal{P}_{p}$ is the set of all nodes $q \neq p$ such that $q$ is in the support of $\varphi_{p}$. For $p \in \mathcal{P}_{h}^{0} \backslash \tilde{\mathcal{P}}_{h}^{0}$, we have to modify the definition. We refer to [16, 58, for the modification of the basis functions in the neighborhood of the boundary $\partial \Omega$. In terms of $\mathbf{X}_{h}$, we can introduce a new operator $\mathbf{Q}^{*}:\left[L^{2}(\Omega)\right]^{2} \longrightarrow \mathbf{X}_{h}$ by

$$
\int_{\Omega} \mathbf{Q}^{*} \mathbf{g} \varphi_{p} \mathrm{~d} x=\int_{\Omega} \mathbf{g} \varphi_{p} \mathrm{~d} x, \quad p \in \mathcal{P}_{h}^{0} .
$$

It is easy to see that $\mathbf{Q}^{*}$ is locally defined. We can write $\mathbf{Q}^{*} \mathbf{g}=\sum_{p \in \mathcal{P}_{h}^{0}} \gamma_{p} \chi_{p}$ with

$$
\gamma_{p}:=\frac{\int_{\Omega} \mathbf{g} \varphi_{p} \mathrm{~d} x}{\int_{\Omega} \varphi_{p} \mathrm{~d} x} .
$$

Associated with $\mathbf{Q}^{*}$ is the operator $\mathbf{Q}:\left[H^{1}(\Omega)\right]^{2} \longrightarrow \mathbf{V}_{h}$ which satisfies the following conditions:

$$
\begin{aligned}
\left.\mathbf{Q} \mathbf{v}\right|_{\Gamma_{C}} & =\mathbf{\Pi} \mathbf{v}, \\
\int_{\Omega} \mathbf{Q} \mathbf{v} \chi_{p} \mathrm{~d} x & =\int_{\Omega} \mathbf{v} \chi_{p} \mathrm{~d} x, \quad p \in \mathcal{P}_{h}^{0}, \\
\mathbf{Q v}(p) & =\mathbf{S v}(p), \quad p \in \mathcal{P}_{h}^{b},
\end{aligned}
$$

where $\mathbf{S}$ is a locally defined Scott-Zhang type operator, see, e.g., 38, preserving the Dirichlet boundary conditions on $\Gamma_{D}$, and $\mathcal{P}_{h}^{b}$ stands for all nodes on $\bar{\Gamma}_{D} \cup \Gamma_{N}$. By (5.30) - (5.32), the operator $\mathbf{Q}$ is uniquely defined. We note that $\mathbf{Q}$ is not well defined for $\left[L^{2}(\Omega)\right]^{2}$ but can be extended to functions with a well-defined trace. This operator is itself a Scott-Zhang type operator, and it can be easily shown that

$$
\begin{aligned}
\|\mathbf{v}-\mathbf{Q v}\|_{0 ; T} & \leq C h_{T}|v|_{1 ; \omega_{T}}, \\
\|\mathbf{v}-\mathbf{Q v}\|_{0 ; e} & \leq C h_{e}^{1 / 2}|v|_{1 ; \omega_{e}},
\end{aligned}
$$

where $\omega_{T}$ includes all elements which share at least one vertex with $T$, and $\omega_{e}$ is the union of all elements having an endpoint of $e$ as vertex. We introduce a 
data-dependent oscillation term

$$
\tilde{\xi}^{2}:=\sum_{T \in \mathcal{T}_{h}} \tilde{\xi}_{T}^{2}, \quad \tilde{\xi}_{T}^{2}:=\frac{h_{T}^{2}}{2 \mu}\left\|\boldsymbol{f}-\mathbf{Q}^{*} \boldsymbol{f}\right\|_{0 ; T}^{2}
$$

and note that $\xi_{T} \leq \tilde{\xi}_{T}$ but that $\tilde{\xi}$ is also of order $h^{2}$ if $\boldsymbol{f} \in\left[H^{1}(\Omega)\right]^{2}$ and that $\tilde{\xi}=0$ if $\boldsymbol{f}$ is constant on $\Omega$.

Theorem 5.1. There exists a constant $C<\infty$ independent of the mesh-size such that

$$
\left\|\boldsymbol{\lambda}-\boldsymbol{\lambda}_{h}\right\|_{-\frac{1}{2} ; \Gamma_{C}} \leq C\left(\left\|\mathbf{u}-\mathbf{u}_{h}\right\|_{a}+\tilde{\xi}\right) .
$$

Proof. We start with the definition of the dual norm and use the definition (2.8) of the mortar projection $\boldsymbol{\Pi}$ and property (5.30) of the operator $\mathbf{Q}$ :

$$
\left\|\boldsymbol{\lambda}-\boldsymbol{\lambda}_{h}\right\|_{-\frac{1}{2} ; \Gamma_{C}}=\sup _{\mathbf{v} \in \mathbf{W}} \frac{b\left(\mathbf{v}, \boldsymbol{\lambda}-\boldsymbol{\lambda}_{h}\right)}{\|\mathbf{v}\|_{\frac{1}{2} ; \Gamma_{C}}}=\sup _{\mathbf{v} \in \mathbf{W}} \frac{b\left(\mathbf{Q v}, \boldsymbol{\lambda}-\boldsymbol{\lambda}_{h}\right)+b(\mathbf{v}-\mathbf{Q v}, \boldsymbol{\lambda})}{\|\mathbf{v}\|_{\frac{1}{2} ; \Gamma_{C}}}
$$

In the following, for simplicity of notation, we denote the harmonic extension of $\mathbf{v} \in \mathbf{W}$ onto $\mathbf{V}_{\mathbf{0}}$ by $\mathbf{v}$. Using the variational equation and the stability of $\mathbf{Q}$, we get

$$
b\left(\mathbf{Q v}, \boldsymbol{\lambda}-\boldsymbol{\lambda}_{h}\right)=a\left(\mathbf{u}_{h}-\mathbf{u}, \mathbf{Q v}\right) \leq C\left\|\mathbf{u}-\mathbf{u}_{h}\right\|_{a}\|\mathbf{Q v}\|_{1} \leq C\left\|\mathbf{u}-\mathbf{u}_{h}\right\|_{a}\|\mathbf{v}\|_{1} .
$$

To bound the second term on the right-hand side, we use the property (5.31), the dual operator $\mathbf{Q}^{*}$ and the stability and the approximation property (5.33) of Q:

$$
\begin{aligned}
b(\mathbf{v}-\mathbf{Q v}, \boldsymbol{\lambda}) & =\boldsymbol{f}(\mathbf{v}-\mathbf{Q v})-a(\mathbf{u}, \mathbf{v}-\mathbf{Q v}) \\
& =\left(\boldsymbol{f}-\mathbf{Q}^{*} \boldsymbol{f}, \mathbf{v}-\mathbf{Q v}\right)_{0}-a\left(\mathbf{u}-\mathbf{u}_{h}, \mathbf{v}-\mathbf{Q v}\right)-a\left(\mathbf{u}_{h}, \mathbf{v}-\mathbf{Q v}\right) \\
& \leq C\left(\tilde{\xi}+\left\|\mathbf{u}-\mathbf{u}_{h}\right\|_{a}\right)\|\mathbf{v}\|_{1}+a\left(\mathbf{u}_{h}, \mathbf{Q v}-\mathbf{v}\right) .
\end{aligned}
$$

Using $b\left(\mathbf{Q v}-\mathbf{v}, \boldsymbol{\lambda}_{h}\right)=b\left(\boldsymbol{\Pi} \mathbf{v}-\mathbf{v}, \boldsymbol{\lambda}_{h}\right)=0$, integration by parts and the approximation property (5.34) give that the last term can be bounded by

$$
\begin{aligned}
a\left(\mathbf{u}_{h}, \mathbf{Q v}-\mathbf{v}\right) & =a\left(\mathbf{u}_{h}, \mathbf{Q v}-\mathbf{v}\right)+b\left(\mathbf{Q v}-\mathbf{v}, \boldsymbol{\lambda}_{h}\right) \\
& \leq C\left(\sum_{e \in \mathcal{E}_{h}^{f}} h_{e}\left\|\left[\boldsymbol{\sigma}\left(\mathbf{u}_{h}\right) \mathbf{n}\right]\right\|_{0 ; e}^{2}+\sum_{e \in \mathcal{E}_{h}^{C}} h_{e}\left\|\boldsymbol{\lambda}_{h}+\boldsymbol{\sigma}\left(\mathbf{u}_{h}\right) \mathbf{n}\right\|_{0 ; e}^{2}\right)^{\frac{1}{2}}\|\mathbf{v}\|_{1}
\end{aligned}
$$


These jump-terms have already been bounded by $C\left(\left\|\mathbf{u}-\mathbf{u}_{h}\right\|_{a}^{2}+\xi^{2}\right)$ in (3.25) and (3.28). Hence we get

$$
b(\mathbf{v}-\mathbf{Q v}, \boldsymbol{\lambda}) \leq C\left(\tilde{\xi}+\left\|\mathbf{u}-\mathbf{u}_{h}\right\|_{a}+\xi\right)\|\mathbf{v}\|_{1} \leq C\left(\left\|\mathbf{u}-\mathbf{u}_{h}\right\|_{a}+\tilde{\xi}\right)\|\mathbf{v}\|_{1} .
$$

Finally, using the continuity of the harmonic extension yields the result.

\section{AFEM ENERGY CONTROL}

The error estimator $\eta$ is quite attractive because it provides upper bounds which tend numerically to one, and no constant depending on the Lamé parameters enters into the upper bound for the error. However, by definition, it is an element-based a posteriori error estimator. Using this type of estimator in combination with an AFEM control-based refinement strategy [10, 25, 43, we have to refine not only a marked element but also all elements in $\mathcal{T}_{T}$. This results from the proof of the upper bound for $\eta_{T}$.

Thus, it is more attractive to work with an error estimator providing a more local upper bound for its contributions. As is well known, the standard residual type error estimator is a good choice from this point of view. Using this idea, we define a second error estimator $\hat{\eta}$ in terms of its edge contributions

$$
\hat{\eta}^{2}:=\sum_{e \in \mathcal{E}_{h}^{-}} \hat{\eta}_{e}^{2}, \quad \hat{\eta}_{e}^{2}:=\frac{h_{e}}{2 \mu}\left\|\left[\boldsymbol{\sigma}\left(\mathbf{u}_{h}\right)\right] \mathbf{n}_{e}\right\|_{0 ; e}^{2}+\frac{h_{e}^{2}}{2 \mu}\left\|\boldsymbol{\Pi}_{0} \boldsymbol{f}\right\|_{0 ; \omega_{e}}^{2},
$$

where $\mathcal{E}_{h}^{-}$denotes all edges not on $\Gamma_{C}$. The natural definition of the jump on the interior edges is extended, as in the last section, to the boundary edges by taking into account the different boundary conditions on $\Gamma_{N}$ and $\Gamma_{D}$. Here $\omega_{e}$ is the union of at most two elements such that $\bar{\omega}_{e}:=\bar{T}_{1} \cup \bar{T}_{2}$ for each interior edge $e=\partial T_{1} \cap \partial T_{2}$ and $\omega_{e}=T$ for each boundary edge $e=\partial T \cap \partial \Omega$. Additionally, the data oscillation term is defined by

$$
\hat{\xi}^{2}:=\sum_{T \in \mathcal{T}_{h}} \hat{\xi}_{T}^{2}, \quad \hat{\xi}_{T}^{2}:=\frac{h_{T}^{2}}{2 \mu}\left\|\boldsymbol{f}-\boldsymbol{\Pi}_{0} \boldsymbol{f}\right\|_{0 ; T}^{2} .
$$

For the AFEM strategy, we consider a sequence of triangulations generated by a special refinement strategy based on the error estimator $\hat{\eta}$. Therefore, the lower index $h$ indicating the mesh-size is replaced by $l$ standing for the actual refinement step; e.g., $\mathcal{T}_{h}$ is replaced by $\mathcal{T}_{l}$. The refinement is carried out by bisection such that all elements in $\mathcal{T}_{l}$ can be written as a union of elements in $\mathcal{T}_{l+1}$.

Each marked edge, i.e., $e \in \mathcal{M}_{l} \subseteq \mathcal{E}_{l}$, is decomposed by the refinement into two subedges of the same length and $T \subset \omega_{e}$ is split into subtriangles such that an interior vertex is obtained; see, e.g., 43 .

As is standard for AFEM strategies, we use a bulk criterion to define the set $\mathcal{M}_{l}$ of marked edges. More precisely, for $\theta \in(0,1)$, we select edges such that

$$
\sum_{e \in \mathcal{M}_{l}} \hat{\eta}_{e}^{2} \geq \theta \hat{\eta}_{l}^{2}
$$

Moreover, we require that

$$
\hat{\xi}_{l+1} \leq \frac{1}{2} \hat{\xi}_{l} .
$$

If this is not fulfilled automatically by the next refinement step controlled by the marked edges, additional elements are refined to satisfy (6.36). We note that for 
many contact problems, $\boldsymbol{f}=\mathbf{0}$ or constant volume forces are considered, and thus (6.36) is automatically guaranteed.

The error estimator $\hat{\eta}$ is a standard residual type error estimator for a variational equality. Moreover, no error term due to the contact boundary $\Gamma_{C}$ enters. Thus it is well known that the local error estimator contribution $\hat{\eta}_{e}, e \in \mathcal{M}_{l}$, can be bounded by

$$
\hat{\eta}_{e}^{2} \leq C\left(\left\|\mathbf{u}_{l+1}-\mathbf{u}_{l}\right\|_{a ; \omega_{e}}^{2}+h_{e}^{2}\left\|\boldsymbol{f}-\boldsymbol{\Pi}_{0} \boldsymbol{f}\right\|_{0 ; \omega_{e}}^{2}\right) ;
$$

see, e.g., 43 for the Laplace operator. Of crucial importance for the proof is that $e \in \mathcal{M}_{l}$ is bisected in the next refinement step and that $T \subseteq \omega_{e}$ is decomposed into several subtriangles having one interior vertex. Then we can work with hierarchical bubble functions having local support and follow the lines of [55].

Using the results of the previous sections, we find that $\hat{\eta}$ also provides, up to data oscillation terms, an upper bound for the discretization error. Theorem 3.6 yields $\left\|\mathbf{u}-\mathbf{u}_{l}\right\|_{a} \leq C\left(\eta_{l}+\hat{\xi}_{l}\right)$; therefore it is sufficient to show $\eta_{l} \leq C\left(\hat{\eta}_{l}+\hat{\xi}_{l}\right)$. The proof of the lower bound of the element contributions $\eta_{T}$ guarantees that

$$
\eta_{T}^{2} \leq C\left(\sum_{e \in \mathcal{E}_{T}^{f}} h_{e}\left\|\mathbf{g}_{e}-\left\{\mathcal{C} \varepsilon\left(\mathbf{u}_{h}\right)\right\} \mathbf{n}_{e}\right\|_{0 ; e}^{2}+\eta_{e}^{2}\right)+\sum_{e \in \mathcal{E}_{T}^{C}} h_{e}\left\|\boldsymbol{\lambda}_{h}+\boldsymbol{\sigma}\left(\mathbf{u}_{h}\right) \mathbf{n}\right\|_{0 ; e}^{2} .
$$

The first term appears in the analysis of equilibrated flux-based error estimators and can be bounded by the residual, and the third term is bounded in (3.28) in terms of $\hat{\eta}$ and $\hat{\xi}$. Thus, we obtain

$$
\eta_{l} \leq C\left(\hat{\eta}_{l}+\hat{\xi}_{l}\right)
$$

and the upper bound follows.

In contrast to the conforming variational equality setting, no Galerkin orthogonality holds for our inequality, and thus $\left\|\mathbf{u}-\mathbf{u}_{l}\right\|_{a}^{2}-\left\|\mathbf{u}-\mathbf{u}_{l+1}\right\|_{a}^{2}$ is not equal to $\left\|\mathbf{u}_{l+1}-\mathbf{u}_{l}\right\|_{a}^{2}$. To overcome this difficulty, we apply the techniques introduced in [15] for the obstacle problem. The new idea of [15] is to consider the energy and not the energy norm. Observing that for each triangulation $\mathcal{T}_{l}$, we have $\mathbf{u}_{l} \in K$, we can work with

$$
\delta_{l}:=J\left(\mathbf{u}_{l}\right)-J(\mathbf{u}) \geq 0 .
$$

The variational inequality is equivalent to a constrained minimization problem, i.e., $J(\mathbf{u}) \leq J(\mathbf{v}), \mathbf{v} \in K$, and in terms of $K_{l} \subset K_{l+1}$, we have

$$
0 \leq \delta_{l+1} \leq \delta_{l} .
$$

The following theorem shows that a stronger result can be obtained.

Theorem 6.1. There exist constants $\rho_{1}, \rho_{2}<1$ and $c_{\xi}, C_{\xi}<\infty$ such that

$$
\begin{aligned}
\delta_{l+1} & \leq \rho_{1} \delta_{l}+c_{\xi} \hat{\xi}_{l}^{2}, \\
\delta_{l+1}+C_{\xi} \hat{\xi}_{l+1}^{2} & \leq \rho_{2}\left(\delta_{l}+C_{\xi} \hat{\xi}_{l}^{2}\right) .
\end{aligned}
$$

Proof. The proof follows the lines of [15] and is straightforward. For the convenience of the reader, we outline the basic steps. We observe that $-b\left(\mathbf{u}, \boldsymbol{\lambda}_{l+1}\right)$ does not have to be greater than or equal to zero, but that we have $-b\left(\mathbf{u}_{l}, \boldsymbol{\lambda}_{l+1}\right) \geq 0$. Then, the 
complementary condition on level $l+1$, the bulk criterion 6.35), Corollary 3.8 and (6.37) yield

$$
\begin{aligned}
\delta_{l}-\delta_{l+1} & =J\left(\mathbf{u}_{l}\right)-J\left(\mathbf{u}_{l+1}\right)=\frac{1}{2}\left\|\mathbf{u}_{l}-\mathbf{u}_{l+1}\right\|_{a}^{2}-b\left(\mathbf{u}_{l}, \boldsymbol{\lambda}_{l+1}\right) \\
& \geq \frac{1}{2}\left\|\mathbf{u}_{l}-\mathbf{u}_{l+1}\right\|_{a}^{2} \geq C \sum_{e \in \mathcal{M}_{l}}\left\|\mathbf{u}_{l}-\mathbf{u}_{l+1}\right\|_{a ; \omega_{e}}^{2} \\
& \geq \sum_{e \in \mathcal{M}_{l}}\left(C \hat{\eta}_{e}^{2}-c h_{e}^{2}\left\|\boldsymbol{f}-\boldsymbol{\Pi}_{0} \boldsymbol{f}\right\|_{0 ; \omega_{e}}^{2}\right) \\
& \geq\left(C \hat{\eta}^{2}-c \hat{\xi}_{l}^{2}\right) \geq\left(C \delta_{l}-c \hat{\xi}_{l}^{2}\right),
\end{aligned}
$$

and thus $\delta_{l+1} \leq(1-C) \delta_{l}+c \hat{\xi}_{l}^{2}$. Moreover, the bulk criteria for the data oscillation term give $\hat{\xi}_{l+1}^{2} \leq \frac{1}{2} \hat{\xi}_{l}^{2}$ and thus $\delta_{l+1}+3 c_{\xi} \hat{\xi}_{l+1}^{2} \leq \rho_{1} \delta_{l}+c_{\xi} \hat{\xi}_{l}^{2}+3 c_{\xi} \hat{\xi}_{l+1}^{2} \leq \rho_{1} \delta_{l}+\frac{5}{2} c_{\xi} \hat{\xi}_{l}^{2} \leq$ $\rho_{2}\left(\delta_{l}+3 c_{\xi} \hat{\xi}_{l}^{2}\right)$.

Remark 6.2. We observe that $\hat{\xi}_{l}=0$ for a constant $\boldsymbol{f}$. In that case, the energy term $\delta_{l}$ is a strictly decreasing function with respect to the refinement level $l$. Moreover, we do not have to create inner nodes in the elements.

\section{Numerical RESUlts FOR AFEM STRATEGy}

In this section, we use the AFEM strategy introduced in Section 6 on Examples 2 and 3 of Section 4 . We use a repeated newest vertex bisection to ensure that an inner point is created for adjacent elements of marked edges. For details about the mesh-refinement process, we refer to [43. As a factor in the bulk criterion (6.35), we choose $\theta=0.3$. Additionally, we compare the influence of different refinement strategies on the estimated error and the energy decay and the two error estimators $\eta, \hat{\eta}$ with an approximated error obtained by an extrapolation technique.

7.1. Square on a circle. We use the same problem setting as depicted in Figure 10 and the same material parameters.
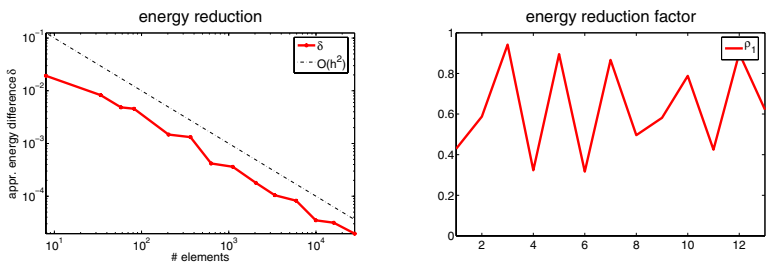

Figure 17. Square on circle (AFEM): Energy reduction and estimated factor $\rho_{1}$.

In the left picture of Figure 17, the energy reduction is plotted. To compute $\delta_{l}$, the exact energy $J(\mathbf{u})$ is approximated using an extrapolation technique. We see that the energy decay is of order $\mathcal{O}\left(h^{2}\right)$. The factor $\rho_{1}$ in Theorem 6.1 can be estimated by $\delta_{l+1} / \delta_{l}$; see the right picture in Figure 17. Here, we obtain a mean value of 0.56 ; moreover, $\rho_{1}$ is bounded by 0.94 . We note that we do not consider volume forces in this example, hence $\hat{\xi}=0$, and we neglect additional terms arising from the nonhomogeneous Neumann boundary conditions. 
7.2. Square on a triangle. We use the same material parameters and problem setting as given in Figure 13. The energy reduction using the AFEM strategy is depicted in Figure 18.
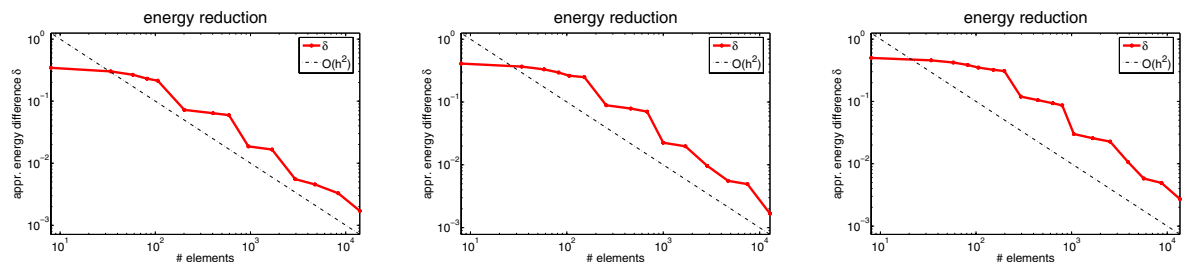

Figure 18. Square on triangle (AFEM): Energy reduction for $\alpha=\pi / 6, \pi / 4, \pi / 3$.

In Figure 19, the estimated reduction factor $\rho_{1}$ is depicted. We get a mean value of 0.72 and maximum of 0.93 for $\alpha=\pi / 6$, mean value of 0.73 and maximum of 0.95 for $\alpha=\pi / 4$ and a mean value of 0.77 and maximum of 0.96 for $\alpha=\pi / 3$. In all our numerical results, we observe that $\rho_{1}$ is oscillating. This can be explained by the "staircase" decrease of the energy differences.
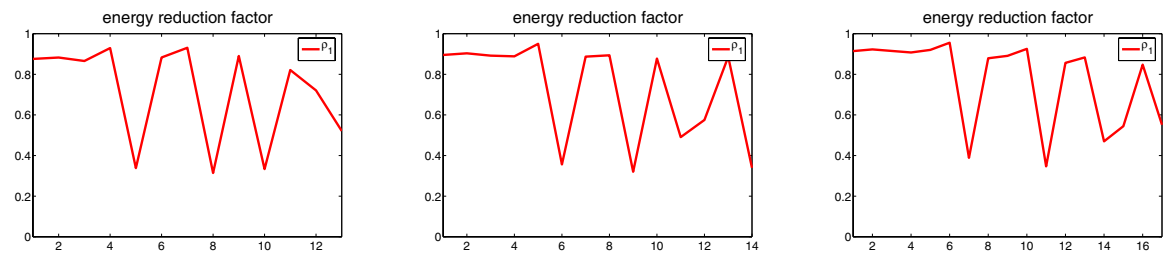

Figure 19. Square on triangle (AFEM): Estimated reduction factor $\rho_{1}$ for $\alpha=\pi / 6, \pi / 4, \pi / 3$.

7.3. Comparison of the refinement strategies. Now we compare the effect of different refinement strategies on the estimated errors and the energy decay. To do so, we chose Example 2 and Example 3 with $\alpha=\pi / 3$.

Depending on the error estimator, the mesh is refined with respect to edges or elements; see Figure 20. We use the residual-based error estimator $\hat{\eta}_{e}$ which results in an edge-based refinement and the error estimators $\eta_{T}$ and $\left(\eta_{T}^{2}+\eta_{e}^{2}\right)^{1 / 2}$ which result in an element-based refinement. For the third error estimator, the corresponding part of $\eta_{C}$ is added to boundary elements, which is motivated by Theorem 3.5. We note that if Assumption 3.1 is not fulfilled for an edge, the corresponding element is marked for refinement. Additionally for each refinement strategy, we use a mesh refinement which leads to an inner point after refinement; see Figure 20 (b).

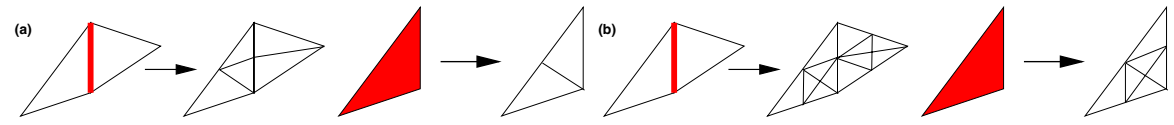

Figure 20. Refinement strategies for edge- and element-based estimators (a) without and (b) with the inner-node property. 
In Figures 21 and 22 the results for Example 2 are given. Here, mesh-sequences generated by different error estimators are compared. On the left side, the energy reduction is given, the value of the error estimator $\eta$ is plotted in the middle and $\hat{\eta}$ is plotted on the right side. Only small differences are observed for the different techniques. We observe that the refinement strategies producing inner nodes perform slightly worse than the others. This can be explained by the more locally refined meshes obtained by the refinement strategies without the inner-node property. Comparing the estimators $\eta_{T}$ and $\left(\eta_{T}^{2}+\eta_{e}^{2}\right)^{1 / 2}$, we see different behavior in the beginning which can be explained by the fact that $\eta$ is not able to estimate the error correctly in this range. Comparing the error estimated by $\eta$ and $\hat{\eta}$, we see that $\eta$ is smaller than the residual estimator $\hat{\eta}$. Asymptotically, however, all error estimators and refinement strategies perform equally well.
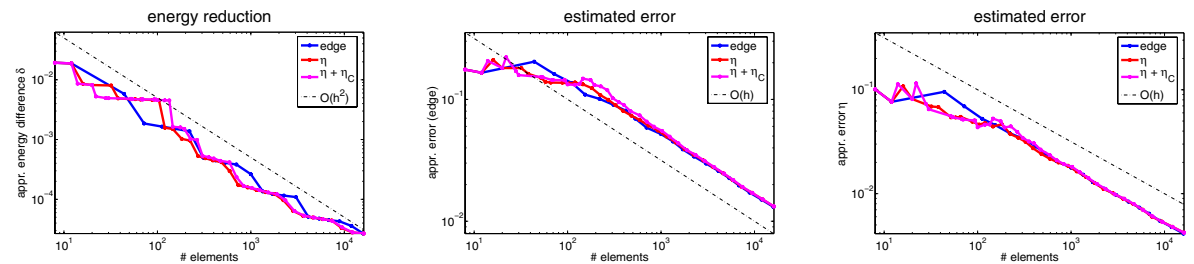

Figure 21. Square on circle: Comparison of different error estimators, refinement strategy (a). Left: energy decay, middle: $\hat{\eta}$, right: $\eta$.
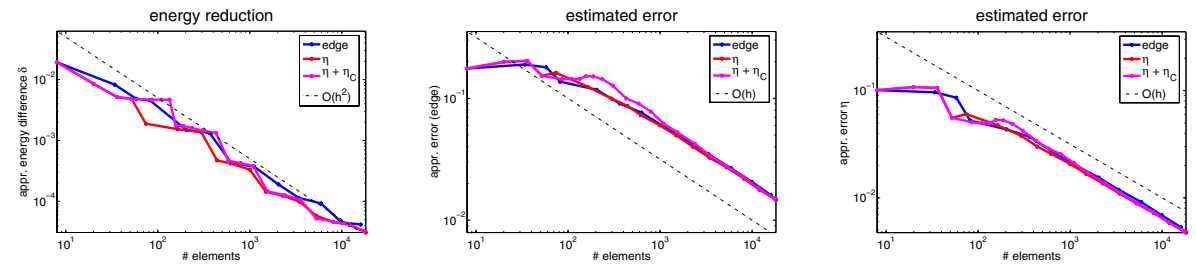

Figure 22. Square on circle: Comparison of different error estimators, refinement strategy (b). Left: energy decay, middle: $\hat{\eta}$, right: $\eta$.

The results for Example 3 are depicted in Figures 23] and 24. Again, we see only small differences between the different strategies.

7.4. Comparison of the error estimators. As we have seen, the influence of the type of refinement on the quantitative and qualitative error and energy decay can be neglected. However, one observes differences between the quantitative values of our error estimators. Therefore, we compare the different error estimators with a higher order error approximation. This approximation is obtained by considering $\left\|\mathbf{u}-\mathbf{u}_{l}\right\|_{a}^{2}=\left(\|\mathbf{u}\|_{a}^{2}-\left\|\mathbf{u}_{l}\right\|_{a}^{2}\right)+2 b\left(\mathbf{u}_{l}, \boldsymbol{\lambda}\right)$, where the first term is approximated using extrapolation techniques and the second term by $b\left(\mathbf{u}_{l}, \boldsymbol{\lambda}_{l_{\max }}\right)$. As error estimators, we consider $\hat{\eta}, \eta$ and $\eta+\eta_{C}$, which corresponds to the upper bound in Theorem 3.5 . 
In Figure 25, the results for Example 2 are given. Here, we only consider meshes that fulfill Assumption 3.1. We see that $\hat{\eta}$ yields an upper bound, but the error is overestimated with a factor of approximately 5 . For the estimator $\eta$, we see that for small meshes the error is even underestimated. $\eta+\eta_{C}$ however gives a good upper bound for the error with a factor of approximately two. Similar results hold for Example 3; see Figure 26. We remark that, due to the singularity, the term $\eta_{C}$ has here a greater influence; see also Figure 16. In all our numerical results, $\eta$ and $\eta+\eta_{C}^{\text {contact }}$ give the best bound.
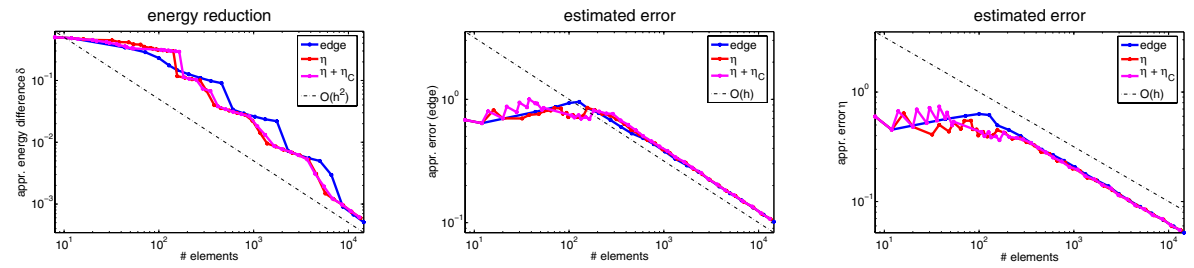

Figure 23. Square on triangle: Comparison of different error estimators, refinement strategy (a). Left: energy decay, middle: $\hat{\eta}$, right: $\eta$.
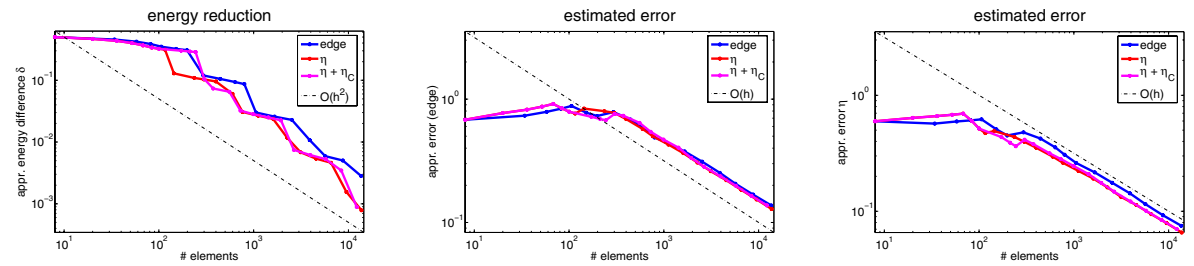

Figure 24. Square on triangle: Comparison of different error estimators, refinement strategy (b). Left: energy decay, middle: $\hat{\eta}$, right: $\eta$.
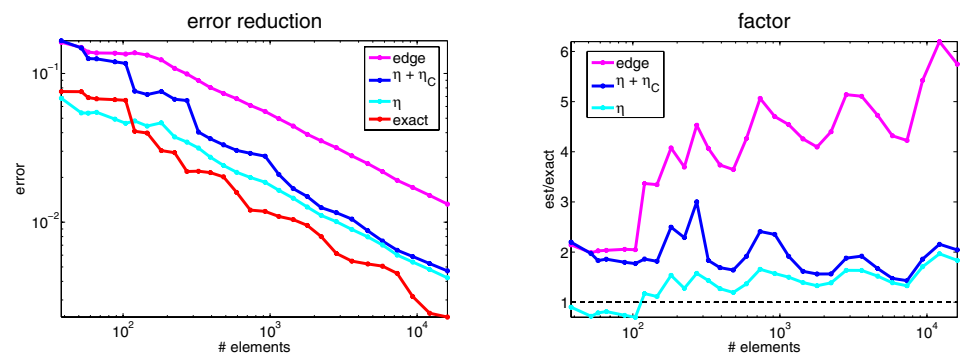

Figure 25. Square on circle: Comparison of error estimators. 

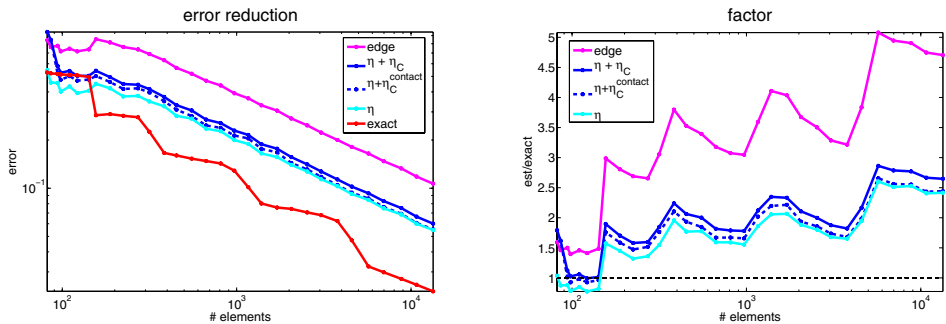

Figure 26. Square on triangle $(\alpha=\pi / 3)$ : Comparison of error estimators.

\section{REFERENCES}

[1] M. Ainsworth, Robust a posteriori error estimation for nonconforming finite element approximation, SIAM J. Numer. Anal., 42 (2005), pp. 2320-234. MR2139395 (2006j:65331)

[2] M. Ainsworth And J. Oden, A posteriori error estimation in finite element analysis, John Wiley and Sons, New York, 2000. MR1885308 (2003b:65001)

[3] M. Ainsworth, J. Oden, and C. Lee, Local a posteriori error estimators for variational inequalities, Numer. Methods Partial Differ. Equations, 9 (1993), pp. 23-33. MR 1193438 (93m:49015)

[4] D. Arnold and R. Winther, Mixed finite elements for elasticity, Numer. Math., 92 (2002), pp. 401-419. MR1930384 (2003i:65103)

[5] I. Babuska AND M. Vogelius, Feedback and adaptive finite element solution of onedimensional boundary value problems, Numer. Math., 44 (1984), pp. 75-102. MR745088 (85k:65070)

[6] I. BABuŠKA And T. Strouboulis, The finite element method and its reliability, Oxford: Clarendon Press, 2001. MR 1857191 (2002k:65001)

[7] F. Ben Belgacem, Numerical simulation of some variational inequalities arisen from unilateral contact problems by the finite element methods, SIAM J. Numer. Anal., 37 (2000), pp. 1198-1216. MR1756422 (2002c:74066)

[8] F. Ben Belgacem, P. Hild, And P. Laborde, Extension of the mortar finite element method to a variational inequality modeling unilateral contact, Mathematical Models \& Methods in Applied Sciences, 9 (1999), pp. 287-303. MR1674556 (99m:73066)

[9] F. Ben Belgacem and Y. Renard, Hybrid finite element methods for the Signorini problem, Mathematics of Computation, 72 (2003), pp. 1117-1145. MR1972730(2004d:65139)

[10] P. Binev, W. Dahmen, And R. DeVore, Adaptive finite element methods with convergence rates, Numer. Math, 97 (2004), pp. 219-268. MR2050077 (2005d:65222)

[11] H. Blum and F. Suttmeier, An adaptive finite element discretisation for a simplified Signorini problem, Calcolo, 37 (2000), pp. 65-77. MR 1819903(2002a:65171)

[12] V. Bostan And W. Han, A posteriori error analysis for finite element solutions of a frictional contact problem, Comput. Methods Appl. Mech. Engrg., 195 (2006), pp. 1252-1274. MR2195300 (2008a:74076)

[13] V. Bostan, W. HAN, AND B. REDDY, A posteriori error estimation and adaptive solution of elliptic variational inequalities of the second kind, Appl. Numer. Math., 52 (2005), pp. 13-38. MR2102908 (2005i:65167)

[14] D. BRAEss, A posteriori error estimators for obstacle problems - another look, Numer. Math., 101 (2005), pp. 523-549. MR2194822 (2006k:65287)

[15] D. Braess, C. Carstensen, And R. Hoppe, Convergence analysis of a conforming adaptive finite element method for an obstacle problem, Numer. Math., 107 (2007), pp. 455-471. MR2336115 (2008f:65208)

[16] D. BRAESS AND W. DAHMEn, The mortar element method revisited-what are the right norms?, in Domain decomposition methods in science and engineering. Papers of the thirteenth international conference on domain decomposition methods, Debit et al., eds., 2002, pp. 27-40. MR2004064

[17] U. BRINK AND E. STEIN, A posteriori error estimation in large-strain elasticity using equilibrated local Neumann problems, Comput. Methods Appl. Mech. Eng., 161 (1998), pp. 77-101. MR1633995(99h:73074) 
[18] G. Buscaglia, R. Duran, E. Fancello, R. Feijoo, and C. Padra, An adaptive finite element approach for frictionless contact problems, Int. J. Numer. Meth. Engng., 50 (2001), pp. 394-418. MR.1807471 (2001j:74085)

[19] C. CARSTEnsen AND S. BARTElS, Each averaging technique yields reliable a posteriori error control in FEM on unstructured grids. I: Low order conforming, nonconforming, and mixed FEM, Math. Comput. 71 (2002), pp. 945-969. MR 1898741(2003e:65212)

[20] C. Carstensen, S. Bartels, and S. Jansche, A posteriori error estimates for nonconforming finite element methods, Numer. Math., 92 (2002), pp. 233-256. MR 1922920|(2003g:65139)

[21] C. Carstensen, P. Causin, and R. Sacco, A posteriori dual-mixed adaptive finite element error control for Lamé and Stokes equations, Numer. Math., 101 (2005), pp. 309-332. MR2195346(2007b:74040)

[22] C. Carstensen, O. Scherf, and P. Wriggers, Adaptive finite elements for elastic bodies in contact, SIAM J. Sci. Comput., 20 (1999), pp. 1605-1626. MR1694675 (2000j:74065)

[23] P. Coorevits, P. Hild, K. Lhalouani, and T. Sassi, Mixed finite element methods for unilateral problems: Convergence analysis and numerical studies, Mathematics of Computation, 71 (2001), pp. 1-25. MR 1862986 (2002g:74043)

[24] E. Dari, R. Duran, C. PADra, AND V. VAMPA, A posteriori error estimators for nonconforming finite element methods, Math. Modelling Numer. Anal., 30 (1996), pp. 385-400. MR:1399496 (97f:65066)

[25] W. DöRFLER, A convergent adaptive algorithm for Poisson's equation, SIAM J. Numer. Anal., 33 (1996), pp. 1106-1124. MR1393904 (97e:65139)

[26] B. Erdmann, M. Frei, R. Hoppe, R. Kornhuber, and U. Wiest, Adaptive finite element methods for variational inequalities, East-West J. Numer. Math., 1 (1993), pp. 165-197. MR.1253634 (94k:65154)

[27] W. HAN, A posteriori error analysis via duality theory. With applications in modeling and numerical approximations, Springer, New York, 2005. MR2101057 (2005k:65003)

[28] J. Haslinger, I. HlaváC̆EK, AND J. NeC̆AS, Numerical methods for unilateral problems in solid mechanics, in Handbook of Numerical Analysis, P. Ciarlet and J.-L. Lions, eds., vol. IV, North-Holland, Amsterdam, 1996, pp. 313-485. MR.1422506

[29] P. HILD, Numerical implementation of two nonconforming finite element methods for unilateral contact, Computer Methods in Applied Mechanics and Engineering, 184 (2000), pp. 99123. MR 1752624 (2000k:74058)

[30] P. Hild AND P. LABORDE, Quadratic finite element methods for unilateral contact problems, Applied Numerical Mathematics, 41 (2002), pp. 410-421. MR.1903172 (2003e:65221)

[31] P. Hild And S. Nicaise, A posteriori error estimations of residual type for Signorini's problem, Numer. Math., 101 (2005), pp. 523-549. MR2194827 (2006j:65347)

[32] R. Hoppe And R. Kornhuber, Adaptive multilevel methods for obstacle problems, SIAM J. Numer. Anal., 31 (1994), pp. 301-323. MR.1276702 (95c:65181)

[33] S. Hüeber And B. Wohlmuth, A primal-dual active set strategy for non-linear multibody contact problems, Computer Methods in Applied Mechanics and Engineering, 194 (2005), pp. 3147-3166. MR2142538 (2005m:74071)

[34] C. Johnson, Adaptive finite element methods for the obstacle problem, Math. Models Methods Appl. Sci., 2 (1992), pp. 483-487. MR1189062 (93k:65093)

[35] D. Kelly, The self-equilibration of residuals and complementary a posteriori error estimates in the finite element method, Int. J. Numer. Methods Eng., 20 (1984), pp. 1491-1506. MR759999 (85h:73039)

[36] D. Kelly AND J. IsLes, Procedures for residual equilibration and local error estimation in the finite element method, Commun. Appl. Numer. Methods, 5 (1989), pp. 497-505.

[37] N. Kikuchi And J. Oden, Contact problems in elasticity: A study of variational inequalities and finite element methods, vol. 8, SIAM Studies in Applied Mathematics, Philadelphia, 1988. MR961258 (89j:73097)

[38] L. SCOTT AND S. ZHANG, Finite element interpolation of nonsmooth functions satisfying boundary conditions, Math. Comp., 54 (1990), pp. 483-493. MR1011446 (90j:65021)

[39] P. LAdevìze AND D. Leguillon, Error estimate procedure in the finite element method and applications, SIAM J. Numer. Anal., 20 (1983), pp. 485-509. MR701093 (84g:65150)

[40] P. LAdevìze And E. Maunder, A general method for recovering equilibrating element tractions, Comput. Methods Appl. Mech. Engrg., 137 (1996), pp. 111-151.

[41] C. Lee AND J. Oden, A posteriori error estimation of h-p finite element approximations of frictional contact problems., Comput. Methods Appl. Mech. Eng., 113 (1994), pp. 11-45. MR.1266922 (95f:73070) 
[42] P. Morin, R. Nochetto, And K. Siebert, Data oscillation and convergence of adaptive FEM, SIAM J. Numer. Anal., 38 (2000), pp. 466-488. MR1770058 (2001g:65157)

[43] _ Convergence of adaptive finite element methods, SIAM Rev., 44 (2002), pp. 631-658. MR 1980447

[44] S. Nicaise, K. Witowski, and B. Wohlmuth, An a posteriori error estimator for the Lamé equation based on $H$ (div)-conforming stress approximations, IMA J. Numer. Anal., 28 (2008), pp. 331-353. MR2401201

[45] R. Nochetто And K. Мекснау, Convergence of adaptive finite element methods for general second order linear elliptic PDEs, SIAM J. Numer. Anal., 43 (2005), pp. 1803-1827. MR2192319 (2006i:65201)

[46] R. Nochetto, A. Schmidt, K. Siebert, and A. Veeser, Pointwise a posteriori error estimates for monotone semi-linear equations, Numer. Math., 104 (2006), pp. 515-538. MR2249676 (2008a:65201)

[47] S. Ohnimus, E. Stein, And E. Walhorn, Local error estimates of FEM for displacements and stresses in linear elasticity by solving local Neumann problems, Int. J. Numer.Meth. Engng., 52 (2001), pp. 727-746. MR.1857633(2002f:74075)

[48] E. Stein And S. Ohnimus, Equilibrium method for postprocessing and error estimation in the finite element method, Comput. Assist. Mech. Eng. Sci., 4 (1997), pp. 645-666.

[49] _ Anisotropic discretization- and model-error estimation in solid mechanics by local Neumann problems, Comput. Methods Appl Mech. Engrg., 176 (1999), pp. 363-385. MR1665353 (2000d:74078)

[50] E. Stein, S. Ohnimus, And E. Walhorn, Adaptive finite element discretization in elasticity and elastoplasticity by global and local error estimators using local Neumann-problems, Zeitschrift f. Angewandte Mathematik und Mechanik, 79 (1999), pp. 147-150.

[51] R. Stevenson, An optimal adaptive finite element method, SIAM J. Numer. Anal., 42 (2005), pp. 2188-2217. MR2139244 (2006e:65226)

[52] _ Optimality of a standard adaptive finite element method, Found. Comput. Math., 7 (2007), pp. 245-269. MR2324418(2008i:65272)

[53] A. VEESER, On a posteriori error estimation for constant obstacle problems, in Numerical methods for viscosity solutions and applications, Falcone, ed., vol. 59 of World Scientific. Ser. Adv. Math. Appl. Sci., Singapore, 2001, pp. 221-234. MR1886715

[54] R. Verfürth, A posteriori error estimation and adaptive mesh-refinement techniques, J. Comp. Appl. Math., 50 (1994), pp. 67-83. MR1284252 (95c:65171)

[55] _ A review of a posteriori error estimation and adaptive mesh-refinement techniques, Wiley-Teubner Series Advances in Numerical Mathematics, Wiley-Teubner, Chichester, Stuttgart, 1996.

[56] - A review of a posteriori error estimation techniques for elasticity problems, Comput. Methods Appl. Mech. Engrg., 176 (1999), pp. 419-440. MR1665355(2000f:74075)

[57] Y. WANG, Preconditioning for the mixed formulation of linear plane elasticity, Ph.D. thesis, Texas A \& M University, 2004.

[58] B. Wohlmuth, Discretization Methods and Iterative Solvers Based on Domain Decomposition, Springer, Berlin, 2001. MR 1820470 (2002c:65231)

[59] _ _ A comparison of dual Lagrange multiplier spaces for mortar finite element discretizations, M ${ }^{2}$ AN Math. Model. Numer. Anal., 36 (2002), pp. 995-1012. MR1958655 (2004b:65193)

[60] - An a posteriori error estimator for two-body contact problems on non-matching meshes, J. Sci Comp., 33 (2007), pp. 25-45. MR2338331(2008f:74084)

[61] P. Wriggers And O. SCherf, Different a posteriori error estimators and indicators for contact problems, Math. Comput. Modelling, 28 (1998), pp. 437-447. MR1648773(99f:73079)

Institute of Applied Analysis and Numerical Simulations (IANS), Universität Stuttgart, Pfaffenwaldring 57, 70569 Stuttgart, Germany

E-mail address: weiss@ians.uni-stuttgart.de

Institute of Applied Analysis and Numerical Simulations (IANS), Universität Stuttgart, Pfaffenwaldring 57, 70569 Stuttgart, Germany

E-mail address: wohlmuth@ians.uni-stuttgart.de 